\author{
DEPARTMENT OF MATHEMATICS \\ UNIVERSITY OF NIJMEGEN The Netherlands
}

\title{
ESTIMATION OF THE MEAN OF A WIENER SHEET
}

Sándor Baran, Gyula Pap, Martien C. A. van Zuijlen

Report No. 0108 (April 2001)

DEPARTMENT OF MATHEMATICS

UNIVERSITY OF NIJMEGEN

Toernooiveld

6525 ED Nijmegen

The Netherlands 


\title{
Estimation of the mean of a Wiener sheet
}

\author{
Sándor Baran, Gyula Pap, Martien C. A. van Zuijlen ${ }^{\dagger}$
}

\begin{abstract}
A standard Wiener sheet shifted by an unknown parameter is observed above a decreasing curve $\Gamma$. By the help of a direct discrete approach and under weaker assumptions than in the paper of Arató, N. M. [3], an explicit formula is derived for the maximum likelihood estimator (MLE) of the shift parameter. The MLE is a weighted linear combination of the values at the endpoints of the curve $\Gamma$ and weighted integrals of the observed process and its normal derivative along the curve $\Gamma$.
\end{abstract}

Keywords. Wiener sheet, Radon-Nikodym derivatives, maximum likelihood estimator, stochastic integral along a curve, weighted integral of the normal derivative of a random process along a curve.

\section{Introduction}

Let $\{W(s, t): s, t \geqslant 0\}$ be a standard Wiener sheet and consider the process $Z(s, t):=W(s, t)+m$, where $m \in \mathbb{R}$ is an unknown parameter. Let $[a, b] \subset(0, \infty)$ and take a continuous, strictly decreasing function $\gamma:[a, b] \rightarrow \mathbb{R}$ with $\gamma(b)>0$. Consider the curve $\Gamma:=\{(s, \gamma(s)): s \in(a, b)\}$ and the set

$$
G:=\left\{(s, t) \in \mathbb{R}^{2}: a \leqslant s \leqslant b, t \geqslant \gamma(s) \text { or } s>b, t \geqslant \gamma(b)\right\} .
$$

Finally, let $\widetilde{G} \subset G$ be a subset containing an $\varepsilon$-strip of $\Gamma$, i.e.,

$$
\left\{(s, t) \in \mathbb{R}^{2}: s \in[a, a+\varepsilon], t \in[\gamma(s), \gamma(a)] \text { or } s \in[a+\varepsilon, b], t \in[\gamma(s), \gamma(s)+\varepsilon]\right\} \subset \widetilde{G}
$$

with some $\varepsilon>0$. Arató, N. M. [3] considered a twice continuously differentiable function $\gamma$ on $[a, b]$ and determined the maximum likelihood estimator (MLE)

\footnotetext{
*Institute of Mathematics and Informatics, University of Debrecen, Pf. 12, H-4010 Debrecen, Hungary

${ }^{\dagger}$ Department of Mathematics, University of Nijmegen, Toernooiveld 1, 6525 ED Nijmegen, The Netherlands

This research has been supported by NWO, The Netherlands (Nederlandse Organisatie voor Wetenschappelijk Onderzoek), by the Hungarian Ministry of Culture and Education under Grant No. FKFP 0121/1999, and by the Hungarian Foundation for Scientific Researchers under Grant No. OTKA-T032361/2000.
} 
of $m$ based on the observation of $\{Z(s, t):(s, t) \in \widetilde{G}\}$ by the help of partial stochastic differential equations. It turned out that the MLE is a weighted linear combination of the values at the endpoints $(a, \gamma(a))$ and $(b, \gamma(b))$ of the curve $\Gamma$ and weighted integrals of the observed process and its normal derivative along the curve $\Gamma$. The purpose of the present paper is to derive the MLE of $m$ under weaker assumptions on the function $\gamma$ applying direct discrete approach instead of partial stochastic differential equations. We remark that the results could have been also derived from the general Feldman-Hajek theorem (see, for example, Kuo [7]), but our direct approach seems to be essentially simpler. Moreover, we note that the results derived in Section 2 for finite samples and also the $L^{2}$-integration theory along a curve as derived in the Sections 4 and 5, might be of independent interest.

1.1 Theorem. Let $[a, b] \subset(0, \infty)$. Suppose that the function $\gamma:[a, b] \rightarrow \mathbb{R}$ is continuous, strictly decreasing with $\gamma(b)>0$ and twice continuously differentiable on $(a, b)$. Moreover, assume that the limits $\gamma^{\prime}(a):=\lim _{s \downarrow a} \gamma^{\prime}(s) \in[-\infty, 0]$ and $\gamma^{\prime}(b):=\lim _{s \uparrow b} \gamma^{\prime}(s) \in[-\infty, 0]$ exist, and

$$
\int_{a}^{b} \frac{\left|\gamma^{\prime}(s) \gamma^{\prime \prime}(s)\right|}{\left(1+\gamma^{\prime}(s)^{2}\right)^{2}} \mathrm{~d} s<\infty
$$

Then the probability measures $\mathrm{P}_{Z}$ and $\mathrm{P}_{W}$, generated on $C(\widetilde{G})$ by the sheets $Z$ and $W$, respectively, are equivalent and the Radon-Nikodym derivative of $\mathrm{P}_{Z}$ with respect to $\mathrm{P}_{W}$ equals

$$
\frac{\mathrm{dP}_{Z}}{\mathrm{dP}_{W}}(Z)=\exp \left\{-\frac{1}{2}\left(A m^{2}-2 \zeta m\right)\right\}
$$

with

$$
\begin{aligned}
A & =\frac{1}{b \gamma(b)}+\int_{a}^{b} \frac{\mathrm{d} s}{s^{2} \gamma(s)}, \\
\zeta & =c_{1} Z(a, \gamma(a))+c_{2} Z(b, \gamma(b))+\int_{\Gamma} y_{1} Z+\int_{\Gamma} y_{2} \partial_{\mathrm{n}} Z,
\end{aligned}
$$

where $\partial_{\mathrm{n}} Z$ denotes the normal derivative of $Z$ and

$$
\begin{gathered}
c_{1}=\left\{\begin{array}{cc}
\frac{1}{a \gamma(a)\left(1+\gamma^{\prime}(a)^{2}\right)} & \text { if } \gamma^{\prime}(a)>-\infty, \\
0 \quad & \text { if } \gamma^{\prime}(a)=-\infty,
\end{array} \quad c_{2}= \begin{cases}\frac{\gamma^{\prime}(b)^{2}}{b \gamma(b)\left(1+\gamma^{\prime}(b)^{2}\right)} & \text { if } \gamma^{\prime}(b)>-\infty, \\
\frac{1}{b \gamma(b)} & \text { if } \gamma^{\prime}(b)=-\infty,\end{cases} \right. \\
y_{1}(s, \gamma(s))=\frac{\gamma^{\prime}(s)\left[\left(\gamma(s) \gamma^{\prime}(s)-s\right)\left(1+\gamma^{\prime}(s)^{2}\right)-2 s \gamma(s) \gamma^{\prime \prime}(s)\right]}{s^{2} \gamma(s)^{2}\left(1+\gamma^{\prime}(s)^{2}\right)^{5 / 2}}, \\
y_{2}(s, \gamma(s))=\frac{\gamma^{\prime}(s)}{s \gamma(s)\left(1+\gamma^{\prime}(s)^{2}\right)} .
\end{gathered}
$$


The maximum likelihood estimator of the shift parameter $m$ based on the observations $\{Z(s, t):(s, t) \in \widetilde{G}\}$ has the form $\widetilde{m}=\zeta / A$ and it has a normal distribution with mean $m$ and variance $1 / A$.

The integrals $\int_{\Gamma} y_{1} Z$ and $\int_{\Gamma} y_{2} \partial_{\mathrm{n}} Z$ are considered as improper $L^{2}$-Riemann integrals, and will be discussed in the Sections 4 and 5, respectively.

1.2 Remark. In order to calculate Radon-Nikodym derivatives we will apply a discrete approximation, i.e., the sample $\{Z(s, t):(s, t) \in \widetilde{G}\}$ will be approximated by finite samples. For a partition $\mathcal{P}: a=s_{1}<s_{2}<\cdots<s_{N-1}<s_{N}=b$ of $[a, b]$, let $|\mathcal{P}|:=\max _{2 \leqslant i \leqslant N}\left(s_{i}-s_{i-1}\right)$. Lemma 2.2 implies that the MLE of $m$ based on any finite sample containing the observations

$$
\left\{Z\left(s_{i}, \gamma\left(s_{i}\right)\right): 1 \leqslant i \leqslant N\right\} \bigcup\left\{Z\left(s_{i}, \gamma\left(s_{i-1}\right)\right): 2 \leqslant i \leqslant N\right\}
$$

and possibly also finitely many observations from

$$
\bigcup_{i=1}^{N}\left\{Z(s, t): s \geqslant s_{i}, t \geqslant \gamma\left(s_{i}\right)\right\}
$$

has the form $\widetilde{m}_{N}=\zeta_{N} / A_{N}$, where

$$
\begin{aligned}
A_{N} & =\sum_{i=1}^{N} \frac{1}{s_{i} \gamma\left(s_{i}\right)}-\sum_{i=2}^{N} \frac{1}{s_{i} \gamma\left(s_{i-1}\right)}, \\
\zeta_{N} & =\sum_{i=1}^{N} \frac{Z\left(s_{i}, \gamma\left(s_{i}\right)\right)}{s_{i} \gamma\left(s_{i}\right)}-\sum_{i=2}^{N} \frac{Z\left(s_{i}, \gamma\left(s_{i-1}\right)\right)}{s_{i} \gamma\left(s_{i-1}\right)} .
\end{aligned}
$$

If $\mathcal{P}_{N}: a=s_{1}^{(N)}<s_{2}^{(N)}<\cdots<s_{N-1}^{(N)}<s_{N}^{(N)}=b, \quad N=1,2, \ldots$ is a sequence of partitions with $\left|\mathcal{P}_{N}\right| \rightarrow 0$ then we shall see in the proof of Theorem 1.1 that $\widetilde{m}_{N} \stackrel{\mathrm{L}^{2}}{\longrightarrow} \widetilde{m}$.

1.3 Example. Let $\Gamma$ is a part of a circle with centre at the origin, i.e., $\gamma(s)=$ $\sqrt{r^{2}-s^{2}}$ with some $r>0$, and $[a, b] \subset(0, r)$. Then

$$
\begin{gathered}
A=\frac{1}{r^{2}}\left(\frac{\sqrt{r^{2}-a^{2}}}{a}+\frac{b}{\sqrt{r^{2}-b^{2}}}\right), \quad c_{1}=\frac{\sqrt{r^{2}-a^{2}}}{r^{2} a}, \quad c_{2}=\frac{b}{r^{2} \sqrt{r^{2}-b^{2}}}, \\
y_{1}\left(s, \sqrt{r^{2}-s^{2}}\right) \equiv 0, \quad y_{2}\left(s, \sqrt{r^{2}-s^{2}}\right) \equiv-\frac{1}{r^{2}},
\end{gathered}
$$

hence

$$
\widetilde{m}=\frac{\frac{\sqrt{r^{2}-a^{2}}}{a} Z\left(a, \sqrt{r^{2}-a^{2}}\right)-\int_{\Gamma} \partial_{\mathrm{n}} Z+\frac{b}{\sqrt{r^{2}-b^{2}}} Z\left(b, \sqrt{r^{2}-b^{2}}\right)}{\frac{\sqrt{r^{2}-a^{2}}}{a}+\frac{b}{\sqrt{r^{2}-b^{2}}}} .
$$


First we derive the Radon-Nikodym derivative of finite samples in Section 2. The proof of Theorem 1.1 is given in Section 3, and it is based on several lemmas given in the Sections 2, 4 and 5.

\section{Radon-Nikodym derivatives of finite samples}

Let $0<s_{1}<s_{2}<\cdots<s_{L}$ and $0<t_{1}<t_{2}<\cdots<t_{M}$ be real numbers and let $1=\lambda_{1}<\lambda_{2}<\cdots<\lambda_{n} \leqslant \lambda_{n+1}=L$ and $1=\mu_{1}<\mu_{2}<\cdots<\mu_{n} \leqslant \mu_{n+1}=M$ be integers. Let

$$
\begin{aligned}
R & :=\left\{(i, j) \in \mathbb{N}^{2}: 1 \leqslant i \leqslant L, 1 \leqslant j \leqslant M\right\}, \\
H & :=\bigcup_{k=1}^{n}\left\{(i, j) \in \mathbb{N}^{2}: \lambda_{k} \leqslant i \leqslant L, \mu_{n-k+1} \leqslant j \leqslant M\right\}, \\
H_{+} & :=\left\{\left(\lambda_{k}, \mu_{n-k+1}\right): k=1, \ldots, n\right\}, \\
H_{-} & :=\left\{\left(\lambda_{k}, \mu_{n-k+2}\right): k=2, \ldots, n\right\}, \\
H_{1} & :=\left\{\left(i, \mu_{k}\right): k=1, \ldots, n, \lambda_{n-k+1}<i \leqslant \lambda_{n-k+2}\right\}, \\
H_{2} & :=\left\{\left(\lambda_{k}, j\right): k=1, \ldots, n, \mu_{n-k+1}<j \leqslant \mu_{n-k+2}\right\}, \\
H_{1,2} & :=\bigcup_{k=1}^{n}\left\{(i, j) \in \mathbb{N}^{2}: \lambda_{k}<i \leqslant L, \mu_{n-k+1}<j \leqslant M\right\}=H \backslash\left(H_{+} \cup H_{-} \cup H_{1} \cup H_{2}\right) . \\
& \quad H_{+}: \bigcirc \quad H_{-}: \square \quad H_{1}: \triangle \quad H_{2}: \nabla \quad H_{1,2}: \diamond
\end{aligned}
$$

Figure 1: An example of the index sets $R, H_{+}, H_{-}, H_{1}, H_{2}$ and $H_{1,2}$ for $n=3$.

2.1 Lemma. The joint density of $\left\{W\left(s_{i}, t_{j}\right):(i, j) \in H\right\}$ has the form

$$
c \cdot f\left(x_{i, j}:(i, j) \in H_{+} \cup H_{-}\right) \cdot g\left(x_{i, j}:(i, j) \in H\right),
$$

where $c$ is a norming constant,

$$
\begin{aligned}
& f\left(x_{i, j}:(i, j) \in H_{+} \cup H_{-}\right)=\exp \left\{-\sum_{(i, j) \in H_{+}} \frac{x_{i, j}^{2}}{2 s_{i} t_{j}}+\sum_{(i, j) \in H_{-}} \frac{x_{i, j}^{2}}{2 s_{i} t_{j}}\right\}, \\
& g\left(x_{i, j}:(i, j) \in H\right)=\exp \left\{-\sum_{(i, j) \in H_{1}} \frac{\left(\Delta_{1} x_{i, j}\right)^{2}}{2\left(\Delta s_{i}\right) t_{j}}-\sum_{(i, j) \in H_{2}} \frac{\left(\Delta_{2} x_{i, j}\right)^{2}}{2 s_{i}\left(\Delta t_{j}\right)}-\sum_{(i, j) \in H_{1,2}} \frac{\left(\Delta_{1} \Delta_{2} x_{i, j}\right)^{2}}{2\left(\Delta s_{i}\right)\left(\Delta t_{j}\right)}\right\}, \\
& \text { where } \Delta_{1} x_{i, j}=x_{i, j}-x_{i-1, j}, \Delta_{2} x_{i, j}=x_{i, j}-x_{i, j-1} \text { and } \Delta s_{i}=s_{i}-s_{i-1} .
\end{aligned}
$$


Proof. The joint density of $\left\{W\left(s_{i}, t_{j}\right):(i, j) \in R\right\}$ equals

$$
c \exp \left\{-\frac{x_{1,1}^{2}}{2 s_{1} t_{1}}-\sum_{i=2}^{L} \frac{\left(\Delta_{1} x_{i, 1}\right)^{2}}{2\left(\Delta s_{i}\right) t_{1}}-\sum_{j=2}^{M} \frac{\left(\Delta_{2} x_{1, j}\right)^{2}}{2 s_{1}\left(\Delta t_{j}\right)}-\sum_{i=2}^{L} \sum_{j=2}^{M} \frac{\left(\Delta_{1} \Delta_{2} x_{i, j}\right)^{2}}{2\left(\Delta s_{i}\right)\left(\Delta t_{j}\right)}\right\},
$$

which is, in fact, a special case of the statement for $n=1,1=\lambda_{1}<\lambda_{2}=L, 1=\mu_{1}<$ $\mu_{2}=M$, hence for $H=R$ with $H_{+}=\{(1,1)\}, H_{-}=\emptyset, H_{1}=\{(i, 1): 2 \leqslant i \leqslant L\}$, $H_{2}=\{(1, j): 2 \leqslant j \leqslant M\}$, and $H_{1,2}=\{(i, j): 2 \leqslant i \leqslant L, 2 \leqslant j \leqslant M\}$. From this we obtain the joint density of $\left\{W\left(s_{i}, t_{j}\right):(i, j) \in H\right\}$ by integrating with respect to the variables $\left\{x_{i, j}:(i, j) \in R \backslash H\right\}$, e.g., in lexicographic order, that is, with respect to the ordering $(i, j) \leqslant(k, \ell) \Longleftrightarrow i \leqslant k, j \leqslant \ell$. In each step we obtain a set which can be represented in the same form as $H$, hence it is sufficient to show that by integrating the formula (2.1) for $H$ with respect to a variable $x_{i, j},(i, j) \in H_{+}$with $(i+1, j) \in H$ and $(i, j+1) \in H$ we obtain the formula $(2.1)$ for $\widehat{H}:=H \backslash\{(i, j)\}$. We have only four terms in the exponent of the formula (2.1) containing the variable $x_{i, j}$, namely

$$
S:=-\frac{x_{i, j}^{2}}{2 s_{i} t_{j}}-\frac{\left(\Delta_{1} x_{i+1, j}\right)^{2}}{2\left(\Delta s_{i+1}\right) t_{j}}-\frac{\left(\Delta_{2} x_{i, j+1}\right)^{2}}{2 s_{i}\left(\Delta t_{j+1}\right)}-\frac{\left(\Delta_{1} \Delta_{2} x_{i+1, j+1}\right)^{2}}{2\left(\Delta s_{i+1}\right)\left(\Delta t_{j+1}\right)} .
$$

This sum can be written in the following form:

$$
\begin{aligned}
S= & -\frac{x_{i, j}^{2}}{2}\left(\frac{1}{s_{i} t_{j}}+\frac{1}{\left(\Delta s_{i+1}\right) t_{j}}+\frac{1}{s_{i}\left(\Delta t_{j+1}\right)}+\frac{1}{\left(\Delta s_{i+1}\right)\left(\Delta t_{j+1}\right)}\right) \\
& +x_{i, j}\left(\frac{x_{i+1, j}}{\left(\Delta s_{i+1}\right) t_{j}}+\frac{x_{i, j+1}}{s_{i}\left(\Delta t_{j+1}\right)}+\frac{x_{i+1, j+1}-x_{i+1, j}-x_{i, j+1}}{\left(\Delta s_{i+1}\right)\left(\Delta t_{j+1}\right)}\right) \\
& -\frac{x_{i+1, j}^{2}}{2\left(\Delta s_{i+1}\right) t_{j}}-\frac{x_{i, j+1}^{2}}{2 s_{i}\left(\Delta t_{j+1}\right)}-\frac{\left(x_{i+1, j+1}-x_{i+1, j}-x_{i, j+1}\right)^{2}}{2\left(\Delta s_{i+1}\right)\left(\Delta t_{j+1}\right)} .
\end{aligned}
$$

The variable $x_{i, j}$ is contained only in the first and second terms. The sum of these terms can be written as follows:

$$
S^{\prime}:=-\frac{s_{i+1} t_{j+1} x_{i, j}^{2}}{2 s_{i} t_{j}\left(\Delta s_{i+1}\right)\left(\Delta t_{j+1}\right)}-\frac{x_{i, j}\left(s_{i} t_{j+1} x_{i+1, j}+s_{i+1} t_{j} x_{i, j+1}-s_{i} t_{j} x_{i+1, j+1}\right)}{s_{i} t_{j}\left(\Delta s_{i+1}\right)\left(\Delta t_{j+1}\right)} .
$$

Forming a full square in the variable $x_{i, j}$, we obtain

$$
\begin{aligned}
S^{\prime}= & -\frac{s_{i+1} t_{j+1}}{2 s_{i} t_{j}\left(\Delta s_{i+1}\right)\left(\Delta t_{j+1}\right)}\left(x_{i, j}-\frac{s_{i}}{s_{i+1}} x_{i+1, j}-\frac{t_{j}}{t_{j+1}} x_{i, j+1}-\frac{s_{i} t_{j}}{s_{i+1} t_{j+1}} x_{i+1, j+1}\right)^{2} \\
& +\frac{\left(s_{i} t_{j+1} x_{i+1, j}+s_{i+1} t_{j} x_{i, j+1}-s_{i} t_{j} x_{i+1, j+1}\right)^{2}}{2 s_{i} t_{j}\left(\Delta s_{i+1}\right)\left(\Delta t_{j+1}\right)} .
\end{aligned}
$$


The first term of $S^{\prime}$ disappears after integrating with respect to the variable $x_{i, j}$, while the second term of $S^{\prime}$ and the remaining last three terms of $S$ can be written as follows:

$$
-\frac{x_{i+1, j}^{2}}{2 s_{i+1} t_{j}}-\frac{x_{i, j+1}^{2}}{2 s_{i} t_{j+1}}+\frac{x_{i+1, j+1}^{2}}{2 s_{i+1} t_{j+1}}-\frac{\left(\Delta_{1} x_{i+1, j+1}\right)^{2}}{2\left(\Delta s_{i+1}\right) t_{j+1}}-\frac{\left(\Delta_{2} x_{i+1, j+1}\right)^{2}}{2 s_{i+1}\left(\Delta t_{j+1}\right)} .
$$

We always have

$$
\begin{gathered}
\widehat{H}_{1}=\left[H_{1} \backslash\{(i+1, j)\}\right] \cup\{(i+1, j+1)\}, \quad \widehat{H}_{2}=\left[H_{2} \backslash\{(i, j+1)\}\right] \cup\{(i+1, j+1)\}, \\
\widehat{H}_{1,2}=H_{1,2} \backslash\{(i+1, j+1)\} .
\end{gathered}
$$

If $x_{i+1, j} \in H_{1}$ and $x_{i, j+1} \in H_{2}$ then

$$
\widehat{H}_{+}=\left[H_{+} \backslash\{(i, j)\}\right] \cup\{(i+1, j),(i, j+1)\}, \quad \widehat{H}_{-}=H_{-} \cup\{(i+1, j+1)\} .
$$

If $x_{i+1, j} \in H_{-}$and $x_{i, j+1} \in H_{2}$ then

$$
\widehat{H}_{+}=\left[H_{+} \backslash\{(i, j)\}\right] \cup\{(i, j+1)\}, \quad \widehat{H}_{-}=\left[H_{-} \backslash\{(i+1, j)\}\right] \cup\{(i+1, j+1)\} .
$$

If $x_{i+1, j} \in H_{1}$ and $x_{i, j+1} \in H_{-}$then

$$
\widehat{H}_{+}=\left[H_{+} \backslash\{(i, j)\}\right] \cup\{(i+1, j)\}, \quad \widehat{H}_{-}=\left[H_{-} \backslash\{(i, j+1)\}\right] \cup\{(i+1, j+1)\} .
$$

If $x_{i+1, j} \in H_{-}$and $x_{i, j+1} \in H_{-}$then

$$
\widehat{H}_{+}=H_{+} \backslash\{(i, j)\}, \quad \widehat{H}_{-}=\left[H_{-} \backslash\{(i+1, j),(i, j+1)\}\right] \cup\{(i+1, j+1)\} .
$$

In all cases we obtain the formula (2.1) for the set $\widehat{H}$, which completes the induction step.

2.2 Lemma. Let $\widetilde{H}$ be a set with $H_{+} \cup H_{-} \subset \widetilde{H} \subset H$. Then the probability measures $\mathrm{P}_{Z}^{\widetilde{H}}$ and $\mathrm{P}_{W}^{\widetilde{H}}$, generated by the random variables $\left\{Z\left(s_{i}, t_{j}\right):(i, j) \in \widetilde{H}\right\}$ and $\left\{W\left(s_{i}, t_{j}\right):(i, j) \in \widetilde{H}\right\}$, respectively, are equivalent and the Radon-Nikodym derivative of $\mathrm{P}_{Z}^{\widetilde{H}}$ with respect to $\mathrm{P}_{W}^{\widetilde{H}}$ equals

$$
\frac{\mathrm{dP}_{Z}^{\widetilde{H}}}{\mathrm{dP}_{W}^{\widetilde{H}}}\left(x_{i, j}:(i, j) \in \widetilde{H}\right)=\exp \left\{-\frac{1}{2}\left(A_{\widetilde{H}} m^{2}-2 y_{\widetilde{H}} m\right)\right\},
$$

where

$$
A_{\widetilde{H}}=\sum_{(i, j) \in H_{+}} \frac{1}{s_{i} t_{j}}-\sum_{(i, j) \in H_{-}} \frac{1}{s_{i} t_{j}}, \quad y_{\widetilde{H}}=\sum_{(i, j) \in H_{+}} \frac{x_{i, j}}{s_{i} t_{j}}-\sum_{(i, j) \in H_{-}} \frac{x_{i, j}}{s_{i} t_{j}} .
$$


The maximum likelihood estimator of the shift parameter $m$ based on the observations $\{Z(s, t):(s, t) \in \widetilde{H}\}$ has the form $\widetilde{m}_{\widetilde{H}}=\zeta_{\widetilde{H}} / A_{\widetilde{H}}$ where

$$
\zeta_{\widetilde{H}}=\sum_{(i, j) \in H_{+}} \frac{Z\left(s_{i}, t_{j}\right)}{s_{i} t_{j}}-\sum_{(i, j) \in H_{-}} \frac{Z\left(s_{i}, t_{j}\right)}{s_{i} t_{j}},
$$

and $\widetilde{m}_{\widetilde{H}}$ has a normal distribution with mean $m$ and variance $1 / A_{\widetilde{H}}$.

Proof. Using Lemma 2.1 we obtain that the joint density of $\left\{W\left(s_{i}, t_{j}\right):(i, j) \in \widetilde{H}\right\}$ is

$$
c f\left(x_{i, j}:(i, j) \in H_{+} \cup H_{-}\right) \int_{\mathbb{R}^{d}} g\left(x_{i, j}:(i, j) \in H\right) \mathrm{d}\left(x_{i, j}:(i, j) \in H \backslash \widetilde{H}\right),
$$

where $d$ denotes the number of points in $H \backslash \widetilde{H}$. Clearly, the joint density of $\left\{Z\left(s_{i}, t_{j}\right):(i, j) \in \widetilde{H}\right\}$ is the product of $c f\left(x_{i, j}-m:(i, j) \in H_{+} \cup H_{-}\right)$and

$$
\begin{aligned}
& \int_{\mathbb{R}^{d}} g\left(x_{i, j}-m:(i, j) \in \widetilde{H} ; x_{i, j}:(i, j) \in H \backslash \widetilde{H}\right) \mathrm{d}\left(x_{i, j}:(i, j) \in H \backslash \widetilde{H}\right) \\
& =\int_{\mathbb{R}^{d}} g\left(z_{i, j}:(i, j) \in \widetilde{H} ; z_{i, j}+m:(i, j) \in H \backslash \widetilde{H}\right) \mathrm{d}\left(z_{i, j}:(i, j) \in H \backslash \widetilde{H}\right) .
\end{aligned}
$$

We have

$$
g\left(z_{i, j}:(i, j) \in \widetilde{H} ; z_{i, j}+m:(i, j) \in H \backslash \widetilde{H}\right)=g\left(z_{i, j}:(i, j) \in H\right),
$$

hence

$$
\frac{\mathrm{dP}_{Z}^{\widetilde{H}}}{\mathrm{dP}_{W}^{\widetilde{H}}}\left(x_{i, j}:(i, j) \in \widetilde{H}\right)=\frac{f\left(x_{i, j}-m:(i, j) \in H_{+} \cup H_{-}\right)}{f\left(x_{i, j}:(i, j) \in H_{+} \cup H_{-}\right)}=\exp \left\{-\frac{1}{2}\left(A_{\widetilde{H}} m^{2}-2 y_{\widetilde{H}} m\right)\right\},
$$

which implies the given form of the MLE of $m$. Clearly, we have $\mathrm{E} \widetilde{m}_{\widetilde{H}}=m$. Finally, introducing the notation

$$
\eta_{\widetilde{H}}=\sum_{(i, j) \in H_{+}} \frac{W\left(s_{i}, t_{j}\right)}{s_{i} t_{j}}-\sum_{(i, j) \in H_{-}} \frac{W\left(s_{i}, t_{j}\right)}{s_{i} t_{j}}
$$

we have $\mathrm{E} \eta_{\widetilde{H}}=0$ and we can show $\mathrm{D}^{2}\left(\eta_{\widetilde{H}}\right)=\mathrm{E} \eta_{\widetilde{H}}^{2}=A_{\widetilde{H}}$ by induction with respect to $n$ :

$$
\begin{aligned}
\mathrm{E} \eta_{\widetilde{H}}^{2} & =\mathrm{E}\left(\left(\frac{W\left(s_{\lambda_{1}}, t_{\mu_{n}}\right)}{s_{\lambda_{1}} t_{\mu_{n}}}-\frac{W\left(s_{\lambda_{2}}, t_{\mu_{n}}\right)}{s_{\lambda_{2}} t_{\mu_{n}}}+\ldots+\frac{W\left(s_{\lambda_{n-1}}, t_{\mu_{2}}\right)}{s_{\lambda_{n-1}} t_{\mu_{2}}}\right)-\left(\frac{W\left(s_{\lambda_{n}}, t_{\mu_{2}}\right)}{s_{\lambda_{n}} t_{\mu_{2}}}-\frac{W\left(s_{\lambda_{n}}, t_{\mu_{1}}\right)}{s_{\lambda_{n}} t_{\mu_{1}}}\right)\right)^{2} \\
& =\left(\frac{1}{s_{\lambda_{1}} t_{\mu_{n}}}-\frac{1}{s_{\lambda_{2}} t_{\mu_{n}}}+\cdots+\frac{1}{s_{\lambda_{n-1}} t_{\mu_{2}}}\right)+\left(\frac{1}{s_{\lambda_{n}} t_{\mu_{2}}}+\frac{1}{s_{\lambda_{n}} t_{\mu_{1}}}-\frac{2}{s_{\lambda_{n}} t_{\mu_{2}}}\right) \\
& =\frac{1}{s_{\lambda_{1}} t_{\mu_{n}}}-\frac{1}{s_{\lambda_{2}} t_{\mu_{n}}}+\cdots+\frac{1}{s_{\lambda_{n-1}} t_{\mu_{2}}}-\frac{1}{s_{\lambda_{n}} t_{\mu_{2}}}+\frac{1}{s_{\lambda_{n}} t_{\mu_{1}}}
\end{aligned}
$$

which implies $\mathrm{D}^{2}\left(\widetilde{m}_{\widetilde{H}}\right)=1 / A_{\widetilde{H}}$.

We note that the factorisation of the density of $\left\{Z\left(s_{i}, t_{j}\right):(i, j) \in \widetilde{H}\right\}$ shows that $\left\{Z\left(s_{i}, t_{j}\right):(i, j) \in H_{+} \cup H_{-}\right\}$is a sufficient statistic for the parameter $m$. 


\section{Proof of Theorem 1.1}

In order to determine Radon-Nikodym derivatives we have developed the following general method based on Section 2.3.2 in Arató [1].

Let $X$ be a separable metric space and let $C(X)$ denote the space of real-valued continuous functions on $X$ with the uniform metric. For a random field $\left\{\xi_{x}: x \in X\right\}$ with continuous trajectories on a probability space $(\Omega, \mathcal{A}, \mathrm{P})$, the induced mapping $\xi: \Omega \rightarrow C(X)$ is measurable (see, e.g., Billingsley [6, Section 1.3]). Let $\mathrm{P}_{\xi}$ denote the probability measure, generated by the process $\xi$ on $(C(X), \mathcal{B}(C(X)))$. For a finite set $X^{\prime}=\left\{x_{1}, \ldots, x_{k}\right\} \subset X$, we denote by $\mathrm{P}_{\xi}^{X^{\prime}}$ the probability measure, generated by the random variable $\xi\left(X^{\prime}\right):=\left(\xi_{x_{1}}, \ldots, \xi_{x_{k}}\right)$ on $\left(\mathbb{R}^{k}, \mathcal{B}\left(\mathbb{R}^{k}\right)\right)$.

3.1 Proposition. Let $\left\{\xi_{x}: x \in X\right\}$ and $\left\{\widetilde{\xi}_{x}: x \in X\right\}$ be random fields with continuous trajectories. Suppose that there exists a measurable function $f: C(X) \rightarrow$ $\mathbb{R}$ such that $\mathrm{E} f(\xi)=1$ and that for any finite set $X_{0} \subset X$, there exists a sequence of finite subsets $X_{n}, n=1,2, \ldots$ with $X_{0} \subset X_{n} \subset X, n=1,2, \ldots$, and with

$$
\frac{\mathrm{dP}_{\widetilde{\xi}}^{X_{n}}}{\mathrm{dP}_{\xi}^{X_{n}}}\left(\xi\left(X_{n}\right)\right) \stackrel{\mathrm{P}}{\longrightarrow} f(\xi) \quad \text { as } n \rightarrow \infty \text {. }
$$

Then $\mathrm{P}_{\widetilde{\xi}}$ is absolutely continuous with respect to $\mathrm{P}_{\xi}$ and $\frac{\mathrm{dP}_{\widetilde{\xi}}}{\mathrm{dP}_{\xi}}=f$.

(The proof can be found in Baran, Pap and Zuijlen [5].)

We will apply Proposition 3.1 for $X=\widetilde{G}$ and for the random fields $\xi=W$ and $\widetilde{\xi}=Z$. First we note that the random variable $\zeta$ can also be expressed in integrals with respect to the Wiener sheet $\{W(s, t): s, t \geqslant 0\}$, namely $\zeta=\eta+m A$, where

$$
\eta=c_{1} W(a, \gamma(a))+c_{2} W(b, \gamma(b))+\int_{\Gamma} y_{1} W+\int_{\Gamma} y_{2} \partial_{\mathrm{n}} W .
$$

This can be seen from the fact that

$$
\zeta=\eta+m\left(c_{1}+c_{2}+\int_{\Gamma} y_{1}\right)
$$

where the integral

$$
\int_{\Gamma} y_{1}=\int_{a}^{b} y_{1}(s, \gamma(s)) \sqrt{1+\gamma^{\prime}(s)^{2}} \mathrm{~d} s
$$

can be calculated using

$$
\frac{\mathrm{d}}{\mathrm{d} s}\left(\frac{1}{s \gamma(s)\left(1+\gamma^{\prime}(s)^{2}\right)}\right)=y_{1}(s, \gamma(s)) \sqrt{1+\gamma^{\prime}(s)^{2}}-\frac{1}{s^{2} \gamma(s)} .
$$

Clearly $\eta$ has a normal distribution with zero mean and at the end of the proof of the theorem we shall see that its variance equals $A$. Hence $\widetilde{m}=\zeta / A$ has a 
normal distribution with mean $m$ and variance $1 / A$. Using the moment generating function of $\eta$ we obtain

$$
\mathrm{E} \exp \left\{-\frac{1}{2}\left(A m^{2}-2 \eta m\right)\right\}=1
$$

The subset $\widetilde{G} \subset G$ contains an $\varepsilon$-strip of $\Gamma$ with some $\varepsilon>0$, hence for a finite subset $\widetilde{G}_{0} \subset \widetilde{G}$, we can put a grid on $\widetilde{G}$, containing the points of $\widetilde{G}_{0}$ and which is sufficiently fine such that its 'lower staircase boundary' is contained in the given $\varepsilon$-strip of $\Gamma$. More exactly, there exists $n, L, M \in \mathbb{N}$ with $n \leqslant L, n \leqslant M$ and real numbers

$$
a=s_{1}<s_{2}<\cdots<s_{n}=b<s_{n+1}<\cdots<s_{L}, \quad \gamma(a)<t_{n+1}<\cdots<t_{M}
$$

such that $\left(\widetilde{G}_{0} \cup\left\{\left(s_{i}, t_{j}\right):(i, j) \in H_{+} \cup H_{-}\right\}\right) \subset\left(\left\{\left(s_{i}, t_{j}\right):(i, j) \in H\right\} \cap \widetilde{G}\right)$, where $t_{i}:=\gamma\left(s_{n-i+1}\right)$ for $i=1, \ldots, n$ and

$$
\begin{gathered}
H:=\bigcup_{k=1}^{n}\left\{(i, j) \in \mathbb{N}^{2}: k \leqslant i \leqslant L, n-k+1 \leqslant j \leqslant M\right\}, \\
H_{+}:=\{(k, n-k+1): k=1, \ldots, n\}, \quad H_{-}:=\{(k, n-k+2): k=2, \ldots, n\} .
\end{gathered}
$$

Using Lemma 2.2 with $\lambda_{k}=\mu_{k}=k$ for $k=1, \ldots, n, \lambda_{n+1}=L, \mu_{n+1}=M$ and with $\widetilde{H}:=H_{+} \cup H_{-} \cup\left\{(i, j):\left(s_{i}, t_{j}\right) \in \widetilde{G}_{0}\right\}$ we conclude that

$$
\frac{\mathrm{dP}_{Z}^{\widetilde{Z}}}{\mathrm{dP}_{W}^{\widetilde{H}}}\left(W\left(s_{i}, t_{j}\right):(i, j) \in \widetilde{H}\right)=\exp \left\{-\frac{1}{2}\left(A_{\widetilde{H}} m^{2}-2 \eta_{\widetilde{H}} m\right)\right\}
$$

where $\mathrm{P}_{Z}^{\widetilde{H}}$ and $\mathrm{P}_{W}^{\widetilde{H}}$ denote the probability measures generated by the random variables $\left\{Z\left(s_{i}, t_{j}\right):(i, j) \in \widetilde{H}\right\}$ and $\left\{W\left(s_{i}, t_{j}\right):(i, j) \in \widetilde{H}\right\}$, respectively, and

$$
A_{\widetilde{H}}=\sum_{i=1}^{n} \frac{1}{s_{i} \gamma\left(s_{i}\right)}-\sum_{i=2}^{n} \frac{1}{s_{i} \gamma\left(s_{i-1}\right)}, \quad \eta_{\widetilde{H}}=\sum_{i=1}^{n} \frac{W\left(s_{i}, \gamma\left(s_{i}\right)\right)}{s_{i} \gamma\left(s_{i}\right)}-\sum_{i=2}^{n} \frac{W\left(s_{i}, \gamma\left(s_{i-1}\right)\right)}{s_{i} \gamma\left(s_{i-1}\right)} .
$$

Hence, by Proposition 3.1, it is sufficient to prove that for any sequence of partitions $\mathcal{P}_{N}: a=s_{1}^{(N)}<s_{2}^{(N)}<\cdots<s_{N}^{(N)}=b, \quad N=1,2, \ldots$ with $\left|\mathcal{P}_{N}\right| \rightarrow 0$ we have $A_{N} \rightarrow A$ and $\eta_{N} \stackrel{\mathrm{L}^{2}}{\longrightarrow} \eta$, where

$$
\begin{aligned}
A_{N} & =\sum_{i=1}^{N} \frac{1}{s_{i}^{(N)} \gamma\left(s_{i}^{(N)}\right)}-\sum_{i=2}^{N} \frac{1}{s_{i}^{(N)} \gamma\left(s_{i-1}^{(N)}\right)}, \\
\eta_{N} & =\sum_{i=1}^{N} \frac{W\left(s_{i}^{(N)}, \gamma\left(s_{i}^{(N)}\right)\right)}{s_{i}^{(N)} \gamma\left(s_{i}^{(N)}\right)}-\sum_{i=2}^{N} \frac{W\left(s_{i}^{(N)}, \gamma\left(s_{i-1}^{(N)}\right)\right)}{s_{i}^{(N)} \gamma\left(s_{i-1}^{(N)}\right)} .
\end{aligned}
$$


Clearly, we have $A_{N} \rightarrow A$ since

$$
A_{N}=\frac{1}{s_{N}^{(N)} \gamma\left(s_{N}^{(N)}\right)}+\sum_{i=2}^{N} \frac{\Delta s_{i}^{(N)}}{s_{i-1}^{(N)} s_{i}^{(N)} \gamma\left(s_{i-1}^{(N)}\right)} \rightarrow \frac{1}{b \gamma(b)}+\int_{a}^{b} \frac{\mathrm{d} s}{s^{2} \gamma(s)}=A .
$$

The aim of the following discussion is to prove convergence $\eta_{N} \stackrel{\mathrm{L}^{2}}{\longrightarrow} \eta$ which completes the proof. Note that $\eta_{N}$ can be decomposed as

$$
\eta_{N}=\eta_{N}^{(1)}+\eta_{N}^{(2)}+\eta_{N}^{(3)}+\eta_{N}^{(4)}+\eta_{N}^{(5)},
$$

where

$$
\begin{aligned}
\eta_{N}^{(1)}= & \sum_{i=2}^{N-1}\left(-\frac{W\left(s_{i+1}^{(N)}, \gamma\left(s_{i}^{(N)}\right)\right)}{s_{i}^{(N)} \gamma\left(s_{i}^{(N)}\right)\left(1+\gamma^{\prime}\left(s_{i}^{(N)}\right)^{-2}\right)}+\frac{W\left(s_{i}^{(N)}, \gamma\left(s_{i}^{(N)}\right)\right)}{s_{i}^{(N)} \gamma\left(s_{i}^{(N)}\right)}\right. \\
& \left.-\frac{W\left(s_{i}^{(N)}, \gamma\left(s_{i-1}^{(N)}\right)\right)}{s_{i}^{(N)} \gamma\left(s_{i}^{(N)}\right)\left(1+\gamma^{\prime}\left(s_{i}^{(N)}\right)^{2}\right)}\right) \\
\eta_{N}^{(2)}= & \sum_{i=2}^{N-1}\left(\frac{1}{s_{i}^{(N)}}-\frac{1}{s_{i+1}^{(N)}}\right) \frac{W\left(s_{i+1}^{(N)}, \gamma\left(s_{i}^{(N)}\right)\right)}{\gamma\left(s_{i}^{(N)}\right)\left(1+\gamma^{\prime}\left(s_{i}^{(N)}\right)^{-2}\right)} \\
\eta_{N}^{(3)}= & \sum_{i=2}^{N-1}\left(\frac{1}{\gamma\left(s_{i}^{(N)}\right)\left(1+\gamma^{\prime}\left(s_{i}^{(N)}\right)^{2}\right)}-\frac{1}{\gamma\left(s_{i-1}^{(N)}\right)\left(1+\gamma^{\prime}\left(s_{i-1}^{(N)}\right)^{2}\right)}\right) \frac{W\left(s_{i}^{(N)}, \gamma\left(s_{i-1}^{(N)}\right)\right)}{s_{i}^{(N)}} \\
\eta_{N}^{(4)}= & \frac{W\left(s_{1}^{(N)}, \gamma\left(s_{1}^{(N)}\right)\right)}{s_{1}^{(N)} \gamma\left(s_{1}^{(N)}\right)}-\frac{W\left(s_{2}^{(N)}, \gamma\left(s_{1}^{(N)}\right)\right)}{s_{2}^{(N)} \gamma\left(s_{1}^{(N)}\right)\left(1+\gamma^{\prime}\left(s_{1}^{(N)}\right)^{-2}\right)} \\
\eta_{N}^{(5)}= & \frac{W\left(s_{N}^{(N)}, \gamma\left(s_{N}^{(N)}\right)\right)}{s_{N}^{(N)} \gamma\left(s_{N}^{(N)}\right)}-\frac{W\left(s_{N}^{(N)}, \gamma\left(s_{N-1}^{(N)}\right)\right)}{s_{N}^{(N)} \gamma\left(s_{N-1}^{(N)}\right)\left(1+\gamma^{\prime}\left(s_{N-1}^{(N)}\right)^{2}\right)}
\end{aligned}
$$

where $\gamma^{\prime}\left(s_{1}^{(N)}\right)^{-2}=\gamma^{\prime}(a)^{-2}:=0$ if $\gamma^{\prime}(a)=-\infty$. (This decomposition can be checked by comparing the coefficients of $W\left(s_{i}^{(N)}, \gamma\left(s_{i}^{(N)}\right)\right)$ and $W\left(s_{i}^{(N)}, \gamma\left(s_{i-1}^{(N)}\right)\right)$.) The convergence $\eta_{N}^{(4)} \stackrel{\mathrm{L}^{2}}{\longrightarrow} c_{1} W(a, \gamma(a))$ can be proved by decomposing the difference:

$$
\begin{aligned}
c_{1} W(a, \gamma(a))-\eta_{N}^{(4)}= & \frac{\Delta_{1} W\left(s_{2}^{(N)}, \gamma\left(s_{1}^{(N)}\right)\right)}{s_{2}^{(N)} \gamma\left(s_{1}^{(N)}\right)\left(1+\gamma^{\prime}\left(s_{1}^{(N)}\right)^{-2}\right)} \\
& -\left(\frac{1}{s_{1}^{(N)}}-\frac{1}{s_{2}^{(N)}}\right) \frac{W\left(s_{1}^{(N)}, \gamma\left(s_{1}^{(N)}\right)\right)}{\gamma\left(s_{1}^{(N)}\right)\left(1+\gamma^{\prime}\left(s_{1}^{(N)}\right)^{-2}\right)}
\end{aligned}
$$


and taking into account that $\Delta_{1} W\left(s_{2}^{(N)}, \gamma\left(s_{1}^{(N)}\right)\right) \stackrel{\mathrm{L}^{2}}{\longrightarrow} 0$. The convergence $\eta_{N}^{(5)} \stackrel{\mathrm{L}^{2}}{\longrightarrow}$ $c_{2} W(b, \gamma(b))$ can be proved in a similar way. The convergence $\eta_{N}^{(1)} \stackrel{\mathrm{L}^{2}}{\longrightarrow} \int_{\Gamma} y_{2} \partial_{\mathrm{n}} W$ follows from Theorem 5.2 since

$$
\begin{aligned}
\eta_{N}^{(1)}=-\sum_{i=2}^{N-1} y_{2}\left(s_{i}^{(N)}, \gamma\left(s_{i}^{(N)}\right)\right)[ & \gamma^{\prime}\left(s_{i}^{(N)}\right)\left\{W\left(s_{i+1}^{(N)}, \gamma\left(s_{i}^{(N)}\right)\right)-W\left(s_{i}^{(N)}, \gamma\left(s_{i}^{(N)}\right)\right)\right\} \\
& \left.+\gamma^{\prime}\left(s_{i}^{(N)}\right)^{-1}\left\{W\left(s_{i}^{(N)}, \gamma\left(s_{i-1}^{(N)}\right)\right)-W\left(s_{i}^{(N)}, \gamma\left(s_{i}^{(N)}\right)\right)\right\}\right],
\end{aligned}
$$

and the function $y_{2}$ satisfies the condition of Theorem 5.2. The convergence $\eta_{N}^{(2)}+$ $\eta_{N}^{(3)} \stackrel{\mathrm{L}^{2}}{\longrightarrow} \int_{\Gamma} y_{1} W$ follows from the Lemmas 4.9 and 4.10 .

Thus we obtained $\eta_{N} \stackrel{\mathrm{L}^{2}}{\longrightarrow} \eta$, which implies $\mathrm{E} \eta_{N}^{2} \stackrel{\mathrm{L}^{2}}{\longrightarrow} \mathrm{E} \eta^{2}$. On the other hand, Lemma 2.2 implies that $\mathrm{E} \eta_{N}^{2}=A_{N} \rightarrow A$, hence we get $\mathrm{E} \eta^{2}=A$. Consequently, the variance of $\eta$ equals $A$, as it was announced at the beginning of the proof of the theorem.

\section{$4 \quad L^{2}-$ Riemann integral of a process along a curve}

First we note the following simple statement about the existence of $L^{2}$-limits (see, e.g., Ash and Gardner [4], Theorem 1.3.2).

4.1 Lemma. Let $\left\{Y_{n}: n \in \mathbb{N}\right\}$ be a sequence of random variables in $L^{2}(\Omega, \mathcal{A}, \mathrm{P})$. Then there exists a random variable $Y \in L^{2}(\Omega, \mathcal{A}, \mathrm{P})$ such that $Y_{n} \stackrel{\mathrm{L}^{2}}{\longrightarrow} Y$ if and only if the limit $\lim _{n, m \rightarrow \infty} \mathrm{E}\left(Y_{n} Y_{m}\right)$ exists. Moreover, if $Y_{n} \stackrel{\mathrm{L}^{2}}{\longrightarrow} Y$ then $\lim _{n, m \rightarrow \infty} \mathrm{E}\left(Y_{n} Y_{m}\right)=$ $\mathrm{E} Y^{2}, \lim _{n \rightarrow \infty} \mathrm{E} Y_{n}=\mathrm{E} Y$, and $\lim _{n \rightarrow \infty} \mathrm{E}\left(X Y_{n}\right)=\mathrm{E}(X Y)$ for all $X \in L^{2}(\Omega, \mathcal{A}, \mathrm{P})$.

Proof. If $Y_{n} \stackrel{\mathrm{L}^{2}}{\longrightarrow} Y$ then $\lim _{n, m \rightarrow \infty} \mathrm{E}\left(Y_{n} Y_{m}\right)=\mathrm{E} Y^{2}$ follows from $\mathrm{E}\left(Y_{n} Y_{m}-Y^{2}\right)=\mathrm{E}\left[\left(Y_{n}-Y\right) Y_{m}\right]+\mathrm{E}\left[\left(Y_{m}-Y\right) Y\right]=\left\langle Y_{n}-Y, Y_{m}\right\rangle_{\mathrm{L}^{2}}+\left\langle Y_{m}-Y, Y\right\rangle_{\mathrm{L}^{2}} \rightarrow 0$, since the inner product product $\langle\cdot, \cdot\rangle_{L^{2}}$ is continuous.

If the limit $\lim _{n, m \rightarrow \infty} \mathrm{E}\left(Y_{n} Y_{m}\right)=: L$ exists then

$$
\left\|Y_{n}-Y_{m}\right\|_{\mathrm{L}^{2}}^{2}=\mathrm{E}\left(Y_{n}-Y_{m}\right)^{2}=\mathrm{E} Y_{n}^{2}-2 \mathrm{E}\left(Y_{n} Y_{m}\right)+\mathrm{E} Y_{m}^{2} \rightarrow L-2 L+L=0
$$

as $n, m \rightarrow \infty$, hence the sequence $Y_{1}, Y_{2}, \ldots$ is a Cauchy-sequence in $L^{2}(\Omega, \mathcal{A}, \mathrm{P})$.

Instead of $Y_{n} \stackrel{\mathrm{L}^{2}}{\longrightarrow} Y$ we sometimes write $Y=\lim _{N \rightarrow \infty} . Y_{N}$.

A process $\{Z(s): s \in[a, b]\}$ is said to be an $L^{2}$-process if $\{Z(s): s \in[a, b]\} \subset$ $L^{2}(\Omega, \mathcal{A}, \mathrm{P})$. We will use $L^{2}$-Riemann integrals of $L^{2}$-processes (see, e.g., Ash and Gardner [4]). 
4.2 Definition. Let $\{Z(s): s \in[a, b]\}$ be an $L^{2}$-process. If there exists a random variable $I$ such that for all sequence of partitions $\mathcal{P}_{N}: a=s_{1}^{(N)}<s_{2}^{(N)}<\cdots<$ $s_{N-1}^{(N)}<s_{N}^{(N)}=b, N=1,2, \ldots$ with $\left|\mathcal{P}_{N}\right| \rightarrow 0$ and for all points $\widetilde{s}_{i}^{(N)} \in\left[s_{i}^{(N)}, s_{i+1}^{(N)}\right]$, $i=1, \ldots, N-1$, we have

$$
\sum_{i=1}^{N-1} Z\left(\widetilde{s}_{i}^{(N)}\right)\left(s_{i+1}^{(N)}-s_{i}^{(N)}\right) \stackrel{\mathrm{L}^{2}}{\longrightarrow} I
$$

then we say that $Z$ is $L^{2}$-Riemann integrable on $[a, b]$ and we write

$$
I=\int_{a}^{b} Z(s) \mathrm{d} s
$$

By the help of Lemma 4.1, it is easy to prove the following lemma.

4.3 Lemma. If the Riemann integral $\int_{a}^{b} \int_{a}^{b} \mathrm{E}[Z(s) Z(t)] \mathrm{d} s \mathrm{~d} t$ exists then $Z$ is $L^{2}-$ Riemann integrable on $[a, b]$. Especially, if the function $(s, t) \mapsto \mathrm{E}[Z(s) Z(t)]$ is continuous on $[a, b] \times[a, b]$ then $Z$ is $L^{2}$-Riemann integrable on $[a, b]$. Moreover, if the processes $Y$ and $Z$ are $L^{2}$-Riemann integrable on $[a, b]$ then

$$
\begin{gathered}
\mathrm{E} \int_{a}^{b} Z(s) \mathrm{d} s=\int_{a}^{b} \mathrm{E} Z(s) \mathrm{d} s, \quad \mathrm{E}\left[Y(s) \int_{a}^{b} Z(t) \mathrm{d} t\right]=\int_{a}^{b} \mathrm{E}[Y(s) Z(t)] \mathrm{d} t \\
\mathrm{E}\left[\int_{a}^{b} Y(s) \mathrm{d} s \int_{a}^{b} Z(t) \mathrm{d} t\right]=\int_{a}^{b} \int_{a}^{b} \mathrm{E}[Y(s) Z(t)] \mathrm{d} s \mathrm{~d} t .
\end{gathered}
$$

Proof. In order to show that $Z$ is $L^{2}$-Riemann integrable, it is sufficient to prove that for all sequence of partitions $\mathcal{P}_{N}: a=s_{1}^{(N)}<s_{2}^{(N)}<\cdots<s_{N-1}^{(N)}<s_{N}^{(N)}=b$, $N=1,2, \ldots$ with $\left|\mathcal{P}_{N}\right| \rightarrow 0$ and for all points $\widetilde{s}_{i}^{(N)} \in\left[s_{i}^{(N)}, s_{i+1}^{(N)}\right], \quad i=1, \ldots, N-1$, the limit $\operatorname{lii.m}_{N \rightarrow \infty} \sum_{i=1}^{N-1} Z\left(\widetilde{s}_{i}^{(N)}\right)\left(s_{i+1}^{(N)}-s_{i}^{(N)}\right)$ exists, since then these limits coincide (because by mixing two such sequences of partitions, the appropriate sequence of sums converges again). We have

$$
\mathrm{E}\left[\sum_{i=1}^{N-1} Z\left(\widetilde{s}_{i}^{(N)}\right)\left(s_{i+1}^{(N)}-s_{i}^{(N)}\right) \sum_{j=1}^{M-1} Z\left(\widetilde{s}_{j}^{(M)}\right)\left(s_{j+1}^{(M)}-s_{j}^{(M)}\right)\right] \rightarrow \int_{a}^{b} \int_{a}^{b} \mathrm{E}[Z(s) Z(t)] \mathrm{d} s \mathrm{~d} t
$$

as $N, M \rightarrow \infty$, hence, by Lemma 4.1, the limit $\operatorname{lik}_{N \rightarrow \infty} \sum_{i=1}^{N-1} Z\left(\widetilde{s}_{i}^{(N)}\right)\left(s_{i+1}^{(N)}-s_{i}^{(N)}\right)$ exists. 
Again by Lemma 4.1 we obtain

$$
\begin{aligned}
\mathrm{E} \int_{a}^{b} Z(s) \mathrm{d} s & =\lim _{N \rightarrow \infty} \mathrm{E}\left[\sum_{i=1}^{N-1} Z\left(\widetilde{s}_{i}^{(N)}\right)\left(s_{i+1}^{(N)}-s_{i}^{(N)}\right)\right]=\int_{a}^{b} \mathrm{E} Z(t) \mathrm{d} t \\
\mathrm{E}\left[Y(s) \int_{a}^{b} Z(t) \mathrm{d} t\right] & =\lim _{N \rightarrow \infty} \mathrm{E}\left[Y(s) \sum_{i=1}^{N-1} Z\left(\widetilde{s}_{i}^{(N)}\right)\left(s_{i+1}^{(N)}-s_{i}^{(N)}\right)\right]=\int_{a}^{b} \mathrm{E}[Y(s) Z(t)] \mathrm{d} t \\
\mathrm{E}\left[\int_{a}^{b} Y(s) \mathrm{d} s \int_{a}^{b} Z(t) \mathrm{d} t\right] & =\lim _{N \rightarrow \infty} \mathrm{E}\left[\sum_{i=1}^{N-1} Y\left(\widetilde{s}_{i}^{(N)}\right)\left(s_{i+1}^{(N)}-s_{i}^{(N)}\right) \sum_{i=1}^{N-1} Z\left(\widetilde{s}_{i}^{(N)}\right)\left(s_{i+1}^{(N)}-s_{i}^{(N)}\right)\right] \\
& =\int_{a}^{b} \int_{a}^{b} \mathrm{E}[Y(s) Z(t)] \mathrm{d} s \mathrm{~d} t .
\end{aligned}
$$

Now we define improper $L^{2}$-Riemann integrals.

4.4 Definition. Let $\{Z(s): s \in(a, b)\}$ be an $L^{2}$-process. Suppose that for all $a<a+\varepsilon<b-\delta<b$, the process $Z$ is $L^{2}$-Riemann integrable on $[a+\varepsilon, b-\delta]$. If $\int_{a+\varepsilon}^{b-\delta} Z(s) \mathrm{d} s$ converges in $L^{2}$ to some random variable $I$ as $\varepsilon \downarrow 0$ and $\delta \downarrow 0$, then we say that the improper $L^{2}-$ Riemann integral $\int_{a}^{b} Z(s) \mathrm{d} s:=I$ exists.

4.5 Lemma. For the existence of the improper $L^{2}$-Riemann integral $\int_{a}^{b} Z(s) \mathrm{d} s$, one of the following conditions is sufficient:

(i) The limit $\lim _{\varepsilon, \varepsilon^{\prime}, \delta, \delta^{\prime} \downarrow 0} \int_{a+\varepsilon}^{b-\delta} \int_{a+\varepsilon^{\prime}}^{b-\delta^{\prime}} \mathrm{E}[Z(s) Z(t)] \mathrm{d} s \mathrm{~d} t$ exists.

(ii) The improper Riemann integral $\int_{a}^{b} \int_{a}^{b} \mathrm{E}[Z(s) Z(t)] \mathrm{d} s \mathrm{~d} t$ exists.

(iii) The function $(s, t) \mapsto \mathrm{E}[Z(s) Z(t)]$ is continuous on $(a, b) \times(a, b)$ and

$$
\int_{a}^{b} \sqrt{\mathrm{E}\left[Z(s)^{2}\right]} \mathrm{d} s<\infty .
$$

Moreover, if the improper $L^{2}$-Riemann integrals $\int_{a}^{b} Y(s) \mathrm{d} s$ and $\int_{a}^{b} Z(t) \mathrm{d} t$ exists then

$$
\begin{gathered}
\mathrm{E} \int_{a}^{b} Z(s) \mathrm{d} s=\int_{a}^{b} \mathrm{E} Z(s) \mathrm{d} s, \quad \mathrm{E}\left[Y(s) \int_{a}^{b} Z(t) \mathrm{d} t\right]=\int_{a}^{b} \mathrm{E}[Y(s) Z(t)] \mathrm{d} t, \\
\mathrm{E}\left[\int_{a}^{b} Y(s) \mathrm{d} s \int_{a}^{b} Z(t) \mathrm{d} t\right]=\lim _{\varepsilon, \delta \downarrow 0} \int_{a+\varepsilon}^{b-\delta} \int_{a+\varepsilon}^{b-\delta} \mathrm{E}[Y(s) Z(t)] \mathrm{d} s \mathrm{~d} t .
\end{gathered}
$$

If the the process $Z$ is $L^{2}$-Riemann integrable on $[a, b]$ then the improper $L^{2}-$ Riemann integral $\int_{a}^{b} Z(s) \mathrm{d} s$ exists and equals the (usual) $L^{2}-$ Riemann integral. 
Proof. By Lemma 4.1, condition (i) is necessary and sufficient. Condition (ii) obviously implies (i). We will prove that condition (iii) also implies (i). For all $\varepsilon_{n} \downarrow 0$ and $\delta_{n} \downarrow 0$, we have

$$
\begin{aligned}
& \left|\int_{a+\varepsilon_{n+k}}^{b-\delta_{n+k}} \int_{a+\varepsilon_{m+\ell}}^{b-\delta_{m+\ell}} \mathrm{E}[Z(s) Z(t)] \mathrm{d} s \mathrm{~d} t-\int_{a+\varepsilon_{n}}^{b-\delta_{n}} \int_{a+\varepsilon_{m}}^{b-\delta_{m}} \mathrm{E}[Z(s) Z(t)] \mathrm{d} s \mathrm{~d} t\right| \\
& \leqslant \iint_{\left[a+\varepsilon_{n+k}, b-\delta_{n+k}\right] \times\left[a+\varepsilon_{m+\ell}, b-\delta_{m+\ell}\right] \backslash\left[a+\varepsilon_{n}, b-\delta_{n}\right] \times\left[a+\varepsilon_{m}, b-\delta_{m}\right]} \sqrt{\mathrm{E}\left[Z(s)^{2}\right] \mathrm{E}\left[Z(t)^{2}\right]} \mathrm{d} s \mathrm{~d} t \\
& \leqslant \int_{\left[a+\varepsilon_{n+k}, b-\delta_{n+k}\right] \backslash\left[a+\varepsilon_{n}, b-\delta_{n}\right]} \sqrt{\mathrm{E}\left[Z(s)^{2}\right]} \mathrm{d} s \int_{a}^{b} \sqrt{\mathrm{E}\left[Z(t)^{2}\right]} \mathrm{d} t \\
& \quad+\int_{a}^{b} \sqrt{\mathrm{E}\left[Z(s)^{2}\right]} \mathrm{d} s \int_{\left[a+\varepsilon_{m+\ell}, b-\delta_{m+\ell}\right] \backslash\left[a+\varepsilon_{m}, b-\delta_{m}\right]} \sqrt{\mathrm{E}\left[Z(t)^{2}\right]} \mathrm{d} t \\
& \quad \rightarrow 0 \quad \text { as } n, m, k, \ell \rightarrow \infty,
\end{aligned}
$$

hence the limit

$$
\lim _{n, m \rightarrow \infty} \int_{a+\varepsilon_{n}}^{b-\delta_{n}} \int_{a+\varepsilon_{m}}^{b-\delta_{m}} \mathrm{E}[Z(s) Z(t)] \mathrm{d} s \mathrm{~d} t
$$

exists, which implies (i).

For further use we prove the following formula of change of variables.

4.6 Lemma. Let $\gamma:[a, b] \rightarrow \mathbb{R}$ be a continuous, strictly increasing or strictly decreasing function, differentiable on $(a, b)$. Then

$$
\int_{\gamma(a)}^{\gamma(b)} Z(t) \mathrm{d} t=\int_{a}^{b} \gamma^{\prime}(s) Z(\gamma(s)) \mathrm{d} s
$$

if one of the improper $L^{2}$-Riemann integrals exists.

Proof. Suppose that $\gamma$ is strictly increasing and the improper $L^{2}$-Riemann integral on the right hand side exists. Let $\mathcal{P}_{N}: \gamma(a)=t_{1}^{(N)}<t_{2}^{(N)}<\cdots<t_{N-1}^{(N)}<$ $t_{N}^{(N)}=\gamma(b), \quad N=1,2, \ldots$, be a sequence of partitions with $\left|\mathcal{P}_{N}\right| \rightarrow 0$, and let $\widetilde{t}_{i}^{(N)} \in\left[t_{i}^{(N)}, t_{i+1}^{(N)}\right], \quad i=1, \ldots, N-1 . \quad$ Let $s_{i}^{(N)}:=\gamma^{-1}\left(t_{i}^{(N)}\right), \quad \widetilde{s}_{i}^{(N)}:=\gamma^{-1}\left(\widetilde{t}_{i}^{(N)}\right)$. Then $\widetilde{\mathcal{P}}_{N}: a=s_{1}^{(N)}<s_{2}^{(N)}<\cdots<s_{N-1}^{(N)}<s_{N}^{(N)}=b, N=1,2, \ldots$, is a sequence of partitions with $\left|\widetilde{\mathcal{P}}_{N}\right| \rightarrow 0$. Moreover, $\widetilde{s}_{i}^{(N)} \in\left[s_{i}^{(N)}, s_{i+1}^{(N)}\right], i=1, \ldots, N-1$, and for all $a<a+\varepsilon<b-\delta<b$, we have

$$
\begin{aligned}
\sum_{\left[t_{i-1}^{(N)}, t_{i}^{(N)}\right] \subset[\gamma(a+\varepsilon), \gamma(b-\delta)]} Z\left(\widetilde{t}_{i}^{(N)}\right)\left(t_{i}^{(N)}-t_{i-1}^{(N)}\right) & =\sum_{\left[_{i-1}^{(N)}, s_{i}^{(N)}\right] \subset[a+\varepsilon, b-\delta]} Z\left(\gamma\left(\widetilde{s}_{i}^{(N)}\right)\right)\left(\gamma\left(s_{i}^{(N)}\right)-\gamma\left(s_{i-1}^{(N)}\right)\right) \\
& \stackrel{\mathrm{L}^{2}}{\longrightarrow} \int_{a+\varepsilon}^{b-\delta} \gamma^{\prime}(s) Z(\gamma(s)) \mathrm{d} s \quad \text { as } \quad N \rightarrow \infty \\
& \stackrel{\mathrm{L}^{2}}{\longrightarrow} \int_{a}^{b} \gamma^{\prime}(s) Z(\gamma(s)) \mathrm{d} s \quad \text { as } \quad \varepsilon, \delta \downarrow 0,
\end{aligned}
$$


hence the improper $L^{2}$-Riemann integral $\int_{\gamma(a)}^{\gamma(b)} Z(t) \mathrm{d} t$ exists and equals the improper $L^{2}$-Riemann integral $\int_{a}^{b} \gamma^{\prime}(s) Z(\gamma(s)) \mathrm{d} s$.

Next we define $L^{2}$-Riemann integral along a curve $\Gamma:=\{(s, \gamma(s)): s \in(a, b)\}$, where $\gamma:(a, b) \rightarrow \mathbb{R}$ is a differentiable function.

4.7 Definition. Let $Z$ be an $L^{2}$-process given along the curve $\Gamma$. Then

$$
\int_{\Gamma} Z:=\int_{a}^{b} Z(s, \gamma(s)) \sqrt{1+\gamma^{\prime}(s)^{2}} \mathrm{~d} s
$$

if this improper $L^{2}$-Riemann integral exists.

4.8 Remark. For all $N=1,2, \ldots$, let $\mathcal{P}_{N}: a=s_{1}^{(N)}<s_{2}^{(N)}<\cdots<s_{N-1}^{(N)}<$ $s_{N}^{(N)}=b$ be a partition such that $\left|\mathcal{P}_{N}\right| \rightarrow 0$ as $N \rightarrow \infty$. Let $\widetilde{s}_{i}^{(N)} \in\left[s_{i}^{(N)}, s_{i+1}^{(N)}\right]$, $i=1, \ldots, N-1$. Suppose that $(s, t) \mapsto \mathrm{E}[Z(s, \gamma(s)) Z(t, \gamma(t))]$ is continuous on $[a, b] \times[a, b]$ and that $\gamma^{\prime}$ can be continuously extended to $[a, b]$. Then the $L^{2}-$ Riemann integral $\int_{\Gamma} Z$ exists and

$$
\begin{aligned}
\int_{\Gamma} Z & =\operatorname{lij}_{N \rightarrow \infty} \sum_{i=1}^{N-1} Z\left(\widetilde{s}_{i}^{(N)}, \gamma\left(\widetilde{s}_{i}^{(N)}\right)\right) \sqrt{1+\gamma^{\prime}\left(\widetilde{s}_{i}^{(N)}\right)^{2}}\left(s_{i+1}^{(N)}-s_{i}^{(N)}\right) \\
& =\operatorname{lij}_{N \rightarrow \infty} \sum_{i=1}^{N-1} Z\left(\widetilde{s}_{i}^{(N)}, \gamma\left(\widetilde{s}_{i}^{(N)}\right)\right) \sqrt{\left(s_{i+1}^{(N)}-s_{i}^{(N)}\right)^{2}+\left(\gamma\left(s_{i+1}^{(N)}\right)-\gamma\left(s_{i}^{(N)}\right)\right)^{2}} \\
& =\operatorname{li}_{N \rightarrow \infty} \sum_{i=1}^{N-1} Z\left(\widetilde{s}_{i}^{(N)}, \gamma\left(\widetilde{s}_{i}^{(N)}\right)\right) \int_{s_{i}^{(N)}}^{s_{i+1}^{(N)}} \sqrt{1+\gamma^{\prime}(s)^{2}} \mathrm{~d} s,
\end{aligned}
$$

since, for example,

$$
\begin{aligned}
& \left\|\sum_{i=1}^{N-1} Z\left(\widetilde{s}_{i}^{(N)}, \gamma\left(\widetilde{s}_{i}^{(N)}\right)\right)\left(\sqrt{1+\gamma^{\prime}\left(\widetilde{s}_{i}^{(N)}\right)^{2}}-\sqrt{1+\left(\frac{\gamma\left(s_{i+1}^{(N)}\right)-\gamma\left(s_{i}^{(N)}\right)}{s_{i+1}^{(N)}-s_{i}^{(N)}}\right)^{2}}\right)\left(s_{i+1}^{(N)}-s_{i}^{(N)}\right)\right\|_{L^{2}} \\
& \leqslant(b-a) \sup _{s \in[a, b]} \sqrt{\mathrm{E}\left[Z(s, \gamma(s))^{2}\right]} \sup _{\substack{a \leqslant u<v \leqslant b \\
v-u \leqslant\left|\mathcal{P}_{N}\right|}}\left|\sqrt{1+\gamma^{\prime}(u)^{2}}-\sqrt{1+\gamma^{\prime}(v)^{2}}\right| \rightarrow 0
\end{aligned}
$$

as $N \rightarrow \infty$.

The function $y_{1}$ in Theorem 1.1 can be decomposed as $y_{1}=y_{1,1}-y_{1,2}$ with $y_{1,1}(s, \gamma(s)):=\frac{\gamma^{\prime}(s)^{2}}{s^{2} \gamma(s)\left(1+\gamma^{\prime}(s)^{2}\right)^{3 / 2}}, \quad y_{1,2}(s, \gamma(s)):=\frac{\gamma^{\prime}(s)\left[1+\gamma^{\prime}(s)^{2}+2 \gamma(s) \gamma^{\prime \prime}(s)\right]}{s \gamma(s)^{2}\left(1+\gamma^{\prime}(s)^{2}\right)^{5 / 2}}$. 
4.9 Lemma. With the notation of the proof of Theorem 1.1 we have

$$
\eta_{N}^{(2)} \stackrel{\mathrm{L}^{2}}{\longrightarrow} \int_{\Gamma} y_{1,1} W
$$

Proof. Since the function

$$
\begin{aligned}
(s, t) & \mapsto \mathrm{E}\left[y_{1,1}(s, \gamma(s)) \sqrt{1+\gamma^{\prime}(s)^{2}} W(s, \gamma(s)) y_{1,1}(t, \gamma(t)) \sqrt{1+\gamma^{\prime}(t)^{2}} W(t, \gamma(t))\right] \\
& =\frac{(s \wedge t) \gamma(s \vee t)}{s^{2} t^{2} \gamma(s) \gamma(t)\left(1+\gamma^{\prime}(s)^{-2}\right)\left(1+\gamma^{\prime}(t)^{-2}\right)}
\end{aligned}
$$

can be continuously extended to $[a, b] \times[a, b]$, the $L^{2}$-Riemann integral

$$
\int_{\Gamma} y_{1,1} W=\int_{a}^{b} \frac{W(s, \gamma(s)) \mathrm{d} s}{s^{2} \gamma(s)\left(1+\gamma^{\prime}(s)^{-2}\right)}
$$

exists and we have

$$
\int_{a}^{b} \frac{W(s, \gamma(s)) \mathrm{d} s}{s^{2} \gamma(s)\left(1+\gamma^{\prime}(s)^{-2}\right)}=\underset{N \rightarrow \infty}{\lim .} S_{N}
$$

where

$$
S_{N}:=\sum_{i=1}^{N-1} \frac{W\left(s_{i}^{(N)}, \gamma\left(s_{i}^{(N)}\right)\right) \Delta s_{i+1}^{(N)}}{\left(s_{i}^{(N)}\right)^{2} \gamma\left(s_{i}^{(N)}\right)\left(1+\gamma^{\prime}\left(s_{i}^{(N)}\right)^{-2}\right)},
$$

and $\mathcal{P}_{N}: a=s_{1}^{(N)}<s_{2}^{(N)}<\cdots<s_{N-1}^{(N)}<s_{N}^{(N)}=b, \quad N=1,2, \ldots$, is a sequence of partitions such that $\left|\mathcal{P}_{N}\right| \rightarrow 0$ as $N \rightarrow \infty$. Hence we have to prove $\eta_{N}^{(2)}-S_{N} \stackrel{\mathrm{L}^{2}}{\longrightarrow} 0$. We have the decomposition $\eta_{N}^{(2)}-S_{N}=S_{N, 1}-S_{N, 2}-X_{N}$, where

$$
\begin{aligned}
S_{N, 1} & :=\sum_{i=2}^{N-1} \frac{\Delta_{1} W\left(s_{i+1}^{(N)}, \gamma\left(s_{i}^{(N)}\right)\right) \Delta s_{i+1}^{(N)}}{s_{i}^{(N)} s_{i+1}^{(N)} \gamma\left(s_{i}^{(N)}\right)\left(1+\gamma^{\prime}\left(s_{i}^{(N)}\right)^{-2}\right)} \\
S_{N, 2} & :=\sum_{i=2}^{N-1}\left(\frac{1}{s_{i}^{(N)}}-\frac{1}{s_{i+1}^{(N)}}\right) \frac{W\left(s_{i}^{(N)}, \gamma\left(s_{i}^{(N)}\right)\right) \Delta s_{i+1}^{(N)}}{s_{i}^{(N)} \gamma\left(s_{i}^{(N)}\right)\left(1+\gamma^{\prime}\left(s_{i}^{(N)}\right)^{-2}\right)} \\
X_{N} & :=\frac{W\left(s_{1}^{(N)}, \gamma\left(s_{1}^{(N)}\right)\right) \Delta s_{2}^{(N)}}{\left(s_{1}^{(N)}\right)^{2} \gamma\left(s_{1}^{(N)}\right)\left(1+\gamma^{\prime}\left(s_{1}^{(N)}\right)^{-2}\right)} .
\end{aligned}
$$

Obviously $X_{N} \stackrel{\mathrm{L}^{2}}{\longrightarrow} 0$. Clearly, $S_{N, 1} \stackrel{\mathrm{L}^{2}}{\longrightarrow} 0$, since

$$
\left\|S_{N, 1}\right\|_{\mathrm{L}^{2}}^{2}=\sum_{i=2}^{N-1} \frac{\gamma\left(s_{i}^{(N)}\right)\left(\Delta s_{i+1}^{(N)}\right)^{3}}{\left[s_{i}^{(N)} s_{i+1}^{(N)} \gamma\left(s_{i}^{(N)}\right)\left(1+\gamma^{\prime}\left(s_{i}^{(N)}\right)^{-2}\right)\right]^{2}} \leqslant \frac{b-a}{a^{4} \gamma(b)}\left|\mathcal{P}_{N}\right|^{2} \rightarrow 0 .
$$


In a similar way $S_{N, 2} \stackrel{\mathrm{L}^{2}}{\longrightarrow} 0$ since

$$
\begin{aligned}
\left\|S_{N, 2}\right\|_{\mathrm{L}^{2}} & \leqslant \sum_{i=2}^{N-1}\left|\frac{1}{s_{i}^{(N)}}-\frac{1}{s_{i+1}^{(N)}}\right| \frac{\left\|W\left(s_{i}^{(N)}, \gamma\left(s_{i}^{(N)}\right)\right)\right\|_{\mathrm{L}^{2} \Delta s_{i+1}^{(N)}}}{s_{i}^{(N)} \gamma\left(s_{i}^{(N)}\right)\left(1+\gamma^{\prime}\left(s_{i}^{(N)}\right)^{-2}\right)} \\
& =\sum_{i=2}^{N-1} \frac{\left(\Delta s_{i+1}^{(N)}\right)^{2} \sqrt{s_{i}^{(N)} \gamma\left(s_{i}^{(N)}\right)}}{\left(s_{i}^{(N)}\right)^{2} s_{i+1}^{(N)} \gamma\left(s_{i}^{(N)}\right)\left(1+\gamma^{\prime}\left(s_{i}^{(N)}\right)^{-2}\right)} \leqslant \frac{b-a}{a^{5 / 2} \gamma(b)^{1 / 2}}\left|\mathcal{P}_{N}\right| \rightarrow 0,
\end{aligned}
$$

hence the proof is complete.

4.10 Lemma. With the notation of the proof of Theorem 1.1, we have

$$
\eta_{N}^{(3)} \stackrel{\mathrm{L}^{2}}{\longrightarrow}-\int_{\Gamma} y_{1,2} W
$$

Proof. Condition (1.1) implies

$$
\begin{gathered}
\int_{a}^{b} \sqrt{\mathrm{E}\left[y_{1,2}(s, \gamma(s))^{2}\left(1+\gamma^{\prime}(s)^{2}\right) W(s, \gamma(s))^{2}\right]} \mathrm{d} s= \\
\int_{a}^{b} \frac{\left|\gamma^{\prime}(s)\left[1+\gamma^{\prime}(s)^{2}+2 \gamma(s) \gamma^{\prime \prime}(s)\right]\right|}{s^{1 / 2} \gamma(s)^{3 / 2}\left(1+\gamma^{\prime}(s)^{2}\right)^{2}} \mathrm{~d} s<\infty,
\end{gathered}
$$

hence by Lemma 4.5 the improper $L^{2}$-Riemann integral

$$
\int_{\Gamma} y_{1,2} W=\int_{a}^{b} \frac{\gamma^{\prime}(s)\left[1+\gamma^{\prime}(s)^{2}+2 \gamma(s) \gamma^{\prime \prime}(s)\right] W(s, \gamma(s)) \mathrm{d} s}{s \gamma(s)^{2}\left(1+\gamma^{\prime}(s)^{2}\right)^{2}}
$$

exists and we have

$$
\int_{\Gamma} y_{1,2} W=\underset{\varepsilon, \delta \downarrow 0}{\lim . \operatorname{li.m}} \lim _{N \rightarrow \infty} S_{N}(\varepsilon, \delta),
$$

where $\mathcal{P}_{N}: a=s_{1}^{(N)}<s_{2}^{(N)}<\cdots<s_{N-1}^{(N)}<s_{N}^{(N)}=b, \quad N=1,2, \ldots$, is a sequence of partitions such that $\left|\mathcal{P}_{N}\right| \rightarrow 0$ as $N \rightarrow \infty$, and

$S_{N}(\varepsilon, \delta):=$

$\sum_{\left[s_{i-1}^{(N)}, s_{i}^{(N)}\right] \subset[a+\varepsilon, b-\delta]} \frac{\gamma^{\prime}\left(s_{i-1}^{(N)}\right)\left[1+\gamma^{\prime}\left(s_{i-1}^{(N)}\right)^{2}+2 \gamma\left(s_{i-1}^{(N)}\right) \gamma^{\prime \prime}\left(s_{i-1}^{(N)}\right)\right] W\left(s_{i-1}^{(N)}, \gamma\left(s_{i-1}^{(N)}\right)\right) \Delta s_{i}^{(N)}}{s_{i-1}^{(N)} \gamma\left(s_{i-1}^{(N)}\right)^{2}\left(1+\gamma^{\prime}\left(s_{i-1}^{(N)}\right)^{2}\right)^{2}}$.

Hence it is sufficient to prove

$$
\lim _{\varepsilon, \delta \downarrow 0} \limsup _{N \rightarrow \infty}\left\|\eta_{N}^{(3)}+S_{N}(\varepsilon, \delta)\right\|_{\mathrm{L}^{2}}=0 .
$$

We have the decomposition $\eta_{N}^{(3)}+S_{N}(\varepsilon, \delta)=-S_{N, 1}(\varepsilon, \delta)+S_{N, 2}(\varepsilon, \delta)+S_{N, 3}(\varepsilon, \delta)$, where 


$$
\begin{aligned}
& S_{N, 1}(\varepsilon, \delta):= \\
& \sum_{\left[s_{i-1}^{(N)}, s_{i}^{(N)}\right] \subset[a+\varepsilon, b-\delta]} \frac{\gamma^{\prime}\left(s_{i-1}^{(N)}\right)\left(1+\gamma^{\prime}\left(s_{i-1}^{(N)}\right)^{2}+2 \gamma\left(s_{i-1}^{(N)}\right) \gamma^{\prime \prime}\left(s_{i-1}^{(N)}\right)\right) \Delta_{1} W\left(s_{i}^{(N)}, \gamma\left(s_{i-1}^{(N)}\right)\right) \Delta s_{i}^{(N)}}{s_{i-1}^{(N)} \gamma\left(s_{i-1}^{(N)}\right)^{2}\left(1+\gamma^{\prime}\left(s_{i-1}^{(N)}\right)^{2}\right)^{2}}, \\
& S_{N, 2}(\varepsilon, \delta):=\sum_{\left[s_{i-1}^{(N)}, s_{i}^{(N)}\right] \subset[a+\varepsilon, b-\delta]} \frac{W\left(s_{i-1}^{(N)}\right)\left(1+\gamma^{\prime}\left(s_{i-1}^{(N)}\right)^{2}\right)}{(N)} \\
& \left(\begin{array}{c}
\frac{\left.\gamma^{\prime}\left(s_{i-1}^{(N)}\right)\left(1+\gamma^{\prime}\left(s_{i-1}^{(N)}\right)^{2}+2 \gamma\left(s_{i-1}^{(N)}\right) \gamma^{\prime \prime}\left(s_{i-1}^{(N)}\right)\right) \Delta s_{i}^{(N)}\right)}{s_{i-1}^{(N)} \gamma\left(s_{i-1}^{(N)}\right)\left(1+\gamma^{\prime}\left(s_{i-1}^{(N)}\right)^{2}\right)} \\
\left.-\frac{\gamma\left(s_{i}^{(N)}\right)\left(1+\gamma^{\prime}\left(s_{i}^{(N)}\right)^{2}\right)-\gamma\left(s_{i-1}^{(N)}\right)\left(1+\gamma^{\prime}\left(s_{i-1}^{(N)}\right)^{2}\right)}{s_{i}^{(N)} \gamma\left(s_{i}^{(N)}\right)\left(1+\gamma^{\prime}\left(s_{i}^{(N)}\right)^{2}\right)}\right)
\end{array}\right.
\end{aligned}
$$

$S_{N, 3}(\varepsilon, \delta):=$

$$
\sum_{\left[s_{i-1}^{(N)}, s_{i}^{(N)}\right] \not \subset[a+\varepsilon, b-\delta]} \frac{\left(\gamma\left(s_{i-1}^{(N)}\right)\left(1+\gamma^{\prime}\left(s_{i-1}^{(N)}\right)^{2}\right)-\gamma\left(s_{i}^{(N)}\right)\left(1+\gamma^{\prime}\left(s_{i}^{(N)}\right)^{2}\right)\right) W\left(s_{i}^{(N)}, \gamma\left(s_{i-1}^{(N)}\right)\right)}{s_{i}^{(N)} \gamma\left(s_{i-1}^{(N)}\right) \gamma\left(s_{i}^{(N)}\right)\left(1+\gamma^{\prime}\left(s_{i-1}^{(N)}\right)^{2}\right)\left(1+\gamma^{\prime}\left(s_{i}^{(N)}\right)^{2}\right)},
$$

Clearly $S_{N, 1}(\varepsilon, \delta) \stackrel{\mathrm{L}^{2}}{\longrightarrow} 0$ for all $a<a+\varepsilon<b-\delta<b$ since

$$
\begin{aligned}
& \quad\left\|S_{N, 1}(\varepsilon, \delta)\right\|_{\mathrm{L}^{2}}^{2} \\
& =\sum_{\left[s_{i-1}^{(N)}, s_{i}^{(N)}\right] \subset[a+\varepsilon, b-\delta]} \frac{\gamma^{\prime}\left(s_{i-1}^{(N)}\right)^{2}\left[1+\gamma^{\prime}\left(s_{i-1}^{(N)}\right)^{2}+2 \gamma\left(s_{i-1}^{(N)}\right) \gamma^{\prime \prime}\left(s_{i-1}^{(N)}\right)\right]^{2} \gamma\left(s_{i-1}^{(N)}\right)\left(\Delta s_{i}^{(N)}\right)^{3}}{\left(s_{i-1}^{(N)}\right)^{2} \gamma\left(s_{i-1}^{(N)}\right)^{4}\left(1+\gamma^{\prime}\left(s_{i-1}^{(N)}\right)^{2}\right)^{4}} \\
& \leqslant \frac{b-a}{a^{2} \gamma(b)^{3}}\left|\mathcal{P}_{N}\right|^{2} \sup _{s \in[a+\varepsilon, b-\delta]} \gamma^{\prime}(s)^{2}\left[1+\gamma^{\prime}(s)^{2}+2 \gamma(s) \gamma^{\prime \prime}(s)\right]^{2}, \\
& \text { hence } S_{N, 1}(\varepsilon, \delta) \stackrel{\mathrm{L}^{2}}{\longrightarrow} 0 \text { as } N \rightarrow \infty \text { for all } a<a+\varepsilon<b-\delta<b .
\end{aligned}
$$

The mean value theorem implies

$$
\begin{aligned}
& \gamma\left(s_{i}^{(N)}\right)\left(1+\gamma^{\prime}\left(s_{i}^{(N)}\right)^{2}\right)-\gamma\left(s_{i-1}^{(N)}\right)\left(1+\gamma^{\prime}\left(s_{i-1}^{(N)}\right)^{2}\right) \\
& =\gamma^{\prime}\left(\widetilde{s}_{i-1}^{(N)}\right)\left[1+\gamma^{\prime}\left(\widetilde{s}_{i-1}^{(N)}\right)^{2}+2 \gamma\left(\widetilde{s}_{i-1}^{(N)}\right) \gamma^{\prime \prime}\left(\widetilde{s}_{i-1}^{(N)}\right)\right] \Delta s_{i}^{(N)}
\end{aligned}
$$


with some $\widetilde{s}_{i-1}^{(N)} \in\left(s_{i-1}^{(N)}, s_{i}^{(N)}\right)$, hence

$$
\begin{aligned}
& \left\|S_{N, 2}(\varepsilon, \delta)\right\|_{\mathrm{L}^{2}} \\
& \leqslant \frac{b-a}{a^{1 / 2} \gamma(b)^{3 / 2}} \sup _{\substack{a+\varepsilon \leqslant u<v \leqslant b-\delta \\
v-u \leqslant\left|\mathcal{P}_{N}\right|}} \mid \gamma^{\prime}(u)\left[1+\gamma^{\prime}(u)^{2}+2 \gamma(u) \gamma^{\prime \prime}(u)\right] \\
& -\gamma^{\prime}(v)\left[1+\gamma^{\prime}(v)^{2}+2 \gamma(v) \gamma^{\prime \prime}(v)\right] \mid \\
& \quad+\frac{(b-a) b^{1 / 2}}{\gamma(b)^{1 / 2}} \sup _{\substack{s \in[a+\varepsilon, b-\delta]\\
}}\left|\gamma^{\prime}(s)\left[1+\gamma^{\prime}(s)^{2}+2 \gamma(s) \gamma^{\prime \prime}(s)\right]\right| \\
& \quad \times \sup _{\substack{a+\varepsilon \leqslant u<v \leqslant b-\delta \\
v-u \leqslant\left|\mathcal{P}_{N}\right|}}\left|\frac{1}{u \gamma(u)\left(1+\gamma^{\prime}(u)^{2}\right)}-\frac{1}{v \gamma(v)\left(1+\gamma^{\prime}(v)^{2}\right)}\right|,
\end{aligned}
$$

which implies $S_{N, 2}(\varepsilon, \delta) \stackrel{\mathrm{L}^{2}}{\longrightarrow} 0$ as $N \rightarrow \infty$ for all $a<a+\varepsilon<b-\delta<b$.

In a similar way

$$
\begin{aligned}
& \left\|S_{N, 3}(\varepsilon, \delta)\right\|_{\mathrm{L}^{2}} \\
& \leqslant \frac{1}{a^{1 / 2} \gamma(b)^{3 / 2}} \sum_{\left[s_{i-1}^{(N)}, s_{i}^{(N)}\right] \not \subset[a+\varepsilon, b-\delta]} \frac{\left|\gamma^{\prime}\left(\widetilde{s}_{i-1}^{(N)}\right)\left[1+\gamma^{\prime}\left(\widetilde{s}_{i-1}^{(N)}\right)^{2}+2 \gamma\left(\widetilde{s}_{i-1}^{(N)}\right) \gamma^{\prime \prime}\left(\widetilde{s}_{i-1}^{(N)}\right)\right]\right|}{\left(1+\gamma^{\prime}\left(s_{i-1}^{(N)}\right)^{2}\right)\left(1+\gamma^{\prime}\left(s_{i}^{(N)}\right)^{2}\right)} \\
& \rightarrow \frac{1}{a^{1 / 2} \gamma(b)^{3 / 2}} \int_{[a, a+\varepsilon] \cup[b-\delta, b]} \frac{\left|\gamma^{\prime}(s)\left[1+\gamma^{\prime}(s)^{2}+2 \gamma(s) \gamma^{\prime \prime}(s)\right]\right|}{\left(1+\gamma^{\prime}(s)^{2}\right)^{2}} \mathrm{~d} s \quad \text { as } N \rightarrow \infty \\
& \rightarrow 0 \quad \text { as } \varepsilon, \delta \downarrow 0,
\end{aligned}
$$

which implies $\lim _{\varepsilon, \delta \downarrow 0} \limsup _{N \rightarrow \infty}\left\|S_{N, 3}(\varepsilon, \delta)\right\|_{\mathrm{L}^{2}} \rightarrow 0$.

\section{Weighted integral of the normal derivative of the Wiener sheet along a curve}

We define $L^{2}$-Riemann integral of the product of a function and the normal derivative of an $L^{2}$-process along a curve $\Gamma:=\{(s, \gamma(s)): s \in(a, b)\}$, where $\gamma:(a, b) \rightarrow \mathbb{R}$ is a differentiable function. We take the normal vector $n_{\Gamma}(s, \gamma(s))$ of the curve $\Gamma$ at the point $(s, \gamma(s)) \in \Gamma$ defined by

$$
n_{\Gamma}(s, \gamma(s)):=\left(-\frac{\gamma^{\prime}(s)}{\sqrt{1+\gamma^{\prime}(s)^{2}}}, \frac{1}{\sqrt{1+\gamma^{\prime}(s)^{2}}}\right)=\left(\psi(s), \sqrt{1-\psi(s)^{2}}\right),
$$

where

$$
\psi(s):=-\frac{\gamma^{\prime}(s)}{\sqrt{1+\gamma^{\prime}(s)^{2}}}, \quad s \in(a, b) .
$$


5.1 Definition. Let $Z$ be an $L^{2}$-process given along the curve $\Gamma$. Let $y: \Gamma \rightarrow \mathbb{R}$. Then

$$
\int_{\Gamma} y \partial_{\mathrm{n}} Z:=\operatorname{li.i.m}_{h \rightarrow 0} \frac{1}{h} \int_{\Gamma} y(\cdot)\left(Z\left(\cdot+h n_{\Gamma}(\cdot)\right)-Z(\cdot)\right)
$$

if the right hand side exists.

Next we give sufficient conditions for the existence of the integral $\int_{\Gamma} y \partial_{\mathrm{n}} W$.

5.2 Theorem. Suppose that $[a, b] \subset(0, \infty)$, the function $\gamma:[a, b] \rightarrow \mathbb{R}$ is continuous, strictly decreasing with $\gamma(b)>0$, the function $\gamma$ is twice continuously differentiable on $(a, b)$, and the limits $\gamma^{\prime}(a):=\lim _{s \downarrow a} \gamma^{\prime}(s) \in[-\infty, 0]$ and $\gamma^{\prime}(b):=\lim _{s \uparrow b} \gamma^{\prime}(s) \in[-\infty, 0]$ exist. Suppose that the functions $s \mapsto \gamma^{\prime}(s) y(s, \gamma(s))$ and $s \mapsto \gamma^{\prime}(s)^{-1} y(s, \gamma(s))$ can be continuously extended to $[a, b]$. Then the integral $\int_{\Gamma} y \partial_{\mathrm{n}} W$ exists and $\int_{\Gamma} y \partial_{\mathrm{n}} W=\underset{N \rightarrow \infty}{\lim .{ }^{\prime}} S(N)$, where

$$
\begin{aligned}
S(N):=-\sum_{i=2}^{N-1} y\left(s_{i}^{(N)}, \gamma\left(s_{i}^{(N)}\right)\right)[ & \gamma^{\prime}\left(s_{i}^{(N)}\right)\left\{W\left(s_{i+1}^{(N)}, \gamma\left(s_{i}^{(N)}\right)\right)-W\left(s_{i}^{(N)}, \gamma\left(s_{i}^{(N)}\right)\right)\right\} \\
& \left.+\gamma^{\prime}\left(s_{i}^{(N)}\right)^{-1}\left\{W\left(s_{i}^{(N)}, \gamma\left(s_{i-1}^{(N)}\right)\right)-W\left(s_{i}^{(N)}, \gamma\left(s_{i}^{(N)}\right)\right)\right\}\right]
\end{aligned}
$$

where $\mathcal{P}_{N}: a=s_{1}^{(N)}<s_{2}^{(N)}<\cdots<s_{N-1}^{(N)}<s_{N}^{(N)}=b, \quad N=1,2, \ldots$, is a sequence of partitions such that $\left|\mathcal{P}_{N}\right| \rightarrow 0$ as $N \rightarrow \infty$.

For the proof of Theorem 5.2 we make use the following lemma.

5.3 Lemma. Let $\mathcal{P}_{N}: a=s_{1}^{(N)}<s_{2}^{(N)}<\cdots<s_{N-1}^{(N)}<s_{N}^{(N)}=b, \quad N=1,2, \ldots$, be a sequence of partitions with $\left|\mathcal{P}_{N}\right| \rightarrow 0$. Let $g:[a, b] \rightarrow \mathbb{R}$ be continuous. Then

$$
\lim _{N, M \rightarrow \infty} S_{N, M}=\lim _{h, h^{\prime} \rightarrow 0} I_{h, h^{\prime}}=\lim _{\substack{N \rightarrow \infty \\ h \rightarrow 0}} J_{N, h}=\int_{a}^{b} \gamma(s) g(s)^{2} \mathrm{~d} s,
$$

where

$$
\begin{array}{r}
S_{N, M}:=\sum_{i=2}^{N-1} \sum_{j=2}^{M-1} g\left(s_{i}^{(N)}\right) g\left(s_{j}^{(M)}\right) \mathrm{E}\left[\left(W\left(s_{i+1}^{(N)}, \gamma\left(s_{i}^{(N)}\right)\right)-W\left(s_{i}^{(N)}, \gamma\left(s_{i}^{(N)}\right)\right)\right)\right. \\
\left.\left(W\left(s_{j+1}^{(M)}, \gamma\left(s_{j}^{(M)}\right)\right)-W\left(s_{j}^{(M)}, \gamma\left(s_{j}^{(M)}\right)\right)\right)\right] \\
I_{h, h^{\prime}}:=\frac{1}{h h^{\prime}} \int_{a}^{b} \int_{a}^{b} g(s) g(t) \mathrm{E}[(W(s+h, \gamma(s))-W(s, \gamma(s))) \\
\left.\left(W\left(t+h^{\prime}, \gamma(t)\right)-W(t, \gamma(t))\right)\right] \mathrm{d} s \mathrm{~d} t
\end{array}
$$




$$
\begin{array}{r}
J_{N, h}:=\frac{1}{h} \sum_{i=2}^{N-1} g\left(s_{i}^{(N)}\right) \int_{a}^{b} \mathrm{E}\left[\left(W\left(s_{i+1}^{(N)}, \gamma\left(s_{i}^{(N)}\right)\right)-W\left(s_{i}^{(N)}, \gamma\left(s_{i}^{(N)}\right)\right)\right)\right. \\
(W(t+h, \gamma(t))-W(t, \gamma(t)))] \mathrm{d} t .
\end{array}
$$

Moreover,

$$
\lim _{N, M \rightarrow \infty} \widetilde{S}_{N, M}=\int_{\gamma(b)}^{\gamma(a)} \gamma^{-1}(t) g\left(\gamma^{-1}(t)\right)^{2} \mathrm{~d} t
$$

where

$$
\begin{array}{r}
\widetilde{S}_{N, M}:=\sum_{i=2}^{N-1} \sum_{j=2}^{M-1} g\left(s_{i}^{(N)}\right) g\left(s_{j}^{(M)}\right) \mathrm{E}\left[\left(W\left(s_{i}^{(N)}, \gamma\left(s_{i-1}^{(N)}\right)\right)-W\left(s_{i}^{(N)}, \gamma\left(s_{i}^{(N)}\right)\right)\right)\right. \\
\left.\left(W\left(s_{j}^{(M)}, \gamma\left(s_{j-1}^{(M)}\right)\right)-W\left(s_{j}^{(M)}, \gamma\left(s_{j}^{(M)}\right)\right)\right)\right] .
\end{array}
$$

Proof of Lemma 5.3. Obviously,

$$
\begin{aligned}
& \mathrm{E}\left[\left(W\left(s_{i+1}^{(N)}, \gamma\left(s_{i}^{(N)}\right)\right)-W\left(s_{i}^{(N)}, \gamma\left(s_{i}^{(N)}\right)\right)\right)\left(W\left(s_{j+1}^{(M)}, \gamma\left(s_{j}^{(M)}\right)\right)-W\left(s_{j}^{(M)}, \gamma\left(s_{j}^{(M)}\right)\right)\right)\right] \\
& =\gamma\left(s_{i}^{(N)} \vee s_{j}^{(M)}\right) \lambda\left(\left[s_{i}^{(N)}, s_{i+1}^{(N)}\right] \cap\left[s_{j}^{(M)}, s_{j+1}^{(M)}\right]\right),
\end{aligned}
$$

where $\lambda$ denotes the Lebesgue measure on $\mathbb{R}$. Clearly,

$$
\begin{aligned}
\int_{a}^{b} \gamma(s) g(s)^{2} \mathrm{~d} s & =\lim _{N \rightarrow \infty} \sum_{i=1}^{N-1} \gamma\left(s_{i}^{(N)}\right) g\left(s_{i}^{(N)}\right)^{2}\left(s_{i+1}^{(N)}-s_{i}^{(N)}\right) \\
& =\lim _{N, M \rightarrow \infty} \sum_{i=1}^{N-1} \sum_{j=1}^{M-1} \gamma\left(s_{i}^{(N)}\right) g\left(s_{i}^{(N)}\right)^{2} \lambda\left(\left[s_{i}^{(N)}, s_{i+1}^{(N)}\right] \cap\left[s_{j}^{(M)}, s_{j+1}^{(M)}\right]\right)
\end{aligned}
$$

since for every $N \in \mathbb{N}$

$$
s_{i+1}^{(N)}-s_{i}^{(N)}=\sum_{j=1}^{M-1} \lambda\left(\left[s_{i}^{(N)}, s_{i+1}^{(N)}\right] \cap\left[s_{j}^{(M)}, s_{j+1}^{(M)}\right]\right) .
$$

Consequently,

$$
\begin{aligned}
& \mid \int_{a}^{b} \gamma(s) g(s)^{2} \mathrm{~d} s \quad-\sum_{i=1}^{N-1} \sum_{j=1}^{M-1} g\left(s_{i}^{(N)}\right) g\left(s_{j}^{(M)}\right) \gamma\left(s_{i}^{(N)} \vee s_{j}^{(M)}\right) \\
& \lambda\left(\left[s_{i}^{(N)}, s_{i+1}^{(N)}\right] \cap\left[s_{j}^{(M)}, s_{j+1}^{(M)}\right]\right) \mid \\
& \leqslant \sup _{\substack{a \leqslant u<v \leqslant b \\
v-u \leqslant \mathcal{P}_{N} \mid}}|\gamma(u \vee v) g(v)-\gamma(u) g(u)| \sum_{i=1}^{N-1} \sum_{j=1}^{M-1}\left|g\left(s_{i}^{(N)}\right)\right| \\
& \lambda\left(\left[s_{i}^{(N)}, s_{i+1}^{(N)}\right] \cap\left[s_{j}^{(M)}, s_{j+1}^{(M)}\right]\right) \rightarrow 0,
\end{aligned}
$$


since the supremum tends to zero and

$$
\begin{gathered}
\sum_{i=1}^{N-1} \sum_{j=1}^{M-1}\left|g\left(s_{i}^{(N)}\right)\right| \lambda\left(\left[s_{i}^{(N)}, s_{i+1}^{(N)}\right] \cap\left[s_{j}^{(M)}, s_{j+1}^{(M)}\right]\right) \\
=\sum_{i=1}^{N-1}\left|g\left(s_{i}^{(N)}\right)\right|\left(s_{i+1}^{(N)}-s_{i}^{(N)}\right) \rightarrow \int_{a}^{b}|g(s)| \mathrm{d} s .
\end{gathered}
$$

By the same method we get

$$
\left|S_{N, M}-\sum_{i=1}^{N-1} \sum_{j=1}^{M-1} g\left(s_{i}^{(N)}\right) g\left(s_{j}^{(M)}\right) \gamma\left(s_{i}^{(N)} \vee s_{j}^{(M)}\right) \lambda\left(\left[s_{i}^{(N)}, s_{i+1}^{(N)}\right] \cap\left[s_{j}^{(M)}, s_{j+1}^{(M)}\right]\right)\right| \rightarrow 0,
$$

thus we obtain $\lim _{N, M \rightarrow \infty} S_{N, M}=\int_{a}^{b} \gamma(s) g(s)^{2} \mathrm{~d} s$.

In a similar way,

$$
\begin{gathered}
\mathrm{E}\left[(W(s+h, \gamma(s))-W(s, \gamma(s)))\left(W\left(t+h^{\prime}, \gamma(t)\right)-W(t, \gamma(t))\right)\right] \\
\quad=\gamma(s \vee t) \lambda\left([s, s+h] \cap\left[t, t+h^{\prime}\right]\right) .
\end{gathered}
$$

We may suppose that $h^{\prime} \leqslant h$ and $a+h^{\prime}<b-h$. Then we have

$$
\int_{a}^{b} \lambda\left([s, s+h] \cap\left[t, t+h^{\prime}\right]\right) \mathrm{d} t= \begin{cases}h h^{\prime}-\left(a+h^{\prime}-s\right)^{2} / 2 & \text { if } s \in\left[a, a+h^{\prime}\right], \\ h h^{\prime} & \text { if } s \in\left[a+h^{\prime}, b-h\right], \\ h h^{\prime}-(s+h-b)^{2} / 2 & \text { if } s \in\left[b-h, b-h+h^{\prime}\right], \\ h h^{\prime}-h^{\prime 2} / 2-h^{\prime}\left(s+h-h^{\prime}-b\right) & \text { if } s \in\left[b-h+h^{\prime}, b\right],\end{cases}
$$

hence $\int_{a}^{b} \gamma(s) g(s)^{2} \mathrm{~d} s=I_{h, h^{\prime}}^{(1)}+I_{h, h^{\prime}}^{(2)}$, where

$$
I_{h, h^{\prime}}^{(1)}=\frac{1}{h h^{\prime}} \int_{a}^{b} \int_{a}^{b} \gamma(s) g(s)^{2} \lambda\left([s, s+h] \cap\left[t, t+h^{\prime}\right]\right) \mathrm{d} s \mathrm{~d} t,
$$

and $\lim _{h, h^{\prime} \rightarrow 0} I_{h, h^{\prime}}^{(2)}=0 . \quad$ We have

$$
\begin{aligned}
& \left|I_{h, h^{\prime}}^{(1)}-\int_{a}^{b} \gamma(s) g(s)^{2} \mathrm{~d} s\right| \\
& \leqslant \sup _{\substack{a \leqslant u<v \leqslant b \\
v-u \leqslant\left|\mathcal{P}_{N}\right|}}|\gamma(u \vee v) g(v)-\gamma(u) g(u)| \frac{1}{h h^{\prime}} \int_{a}^{b} \int_{a}^{b}|g(s)| \lambda\left([s, s+h] \cap\left[t, t+h^{\prime}\right]\right) \mathrm{d} s \mathrm{~d} t \rightarrow 0,
\end{aligned}
$$

since

$$
\frac{1}{h h^{\prime}} \int_{a}^{b} \int_{a}^{b}|g(s)| \lambda\left([s, s+h] \cap\left[t, t+h^{\prime}\right]\right) \mathrm{d} s \mathrm{~d} t=\int_{a}^{b}|g(s)| \mathrm{d} s+I_{h, h^{\prime}}^{(3)}
$$


where $\lim _{h, h^{\prime} \rightarrow 0} I_{h, h^{\prime}}^{(3)}=0$.

The statement $\lim _{\substack{N \rightarrow \infty \\ h \rightarrow 0}} J_{N, h}=\int_{a}^{b} \gamma(s) g(s)^{2} \mathrm{~d} s$ can be proved similarly.

To prove the last statement, we introduce the notation $t_{i}^{(N)}:=\gamma\left(s_{N+1-i}^{(N)}\right)$. We have

$$
\begin{gathered}
\widetilde{S}_{N, M}=\sum_{i=2}^{N-1} \sum_{j=2}^{M-1} g\left(\gamma^{-1}\left(t_{N+1-i}^{(N)}\right)\right) g\left(\gamma^{-1}\left(t_{M+1-j}^{(M)}\right)\right) \gamma^{-1}\left(t_{N+1-i}^{(N)} \vee t_{M+1-j}^{(M)}\right) \\
\lambda\left(\left[t_{N+1-i}^{(N)}, t_{N+2-i}^{(N)}\right] \cap\left[s_{M+1-j}^{(M)}, t_{M+2-j}^{(M)}\right]\right) .
\end{gathered}
$$

Now we can use the first statement of the lemma for the curve $\gamma^{-1}:[\gamma(b), \gamma(a)] \rightarrow \mathbb{R}$ and for the continuous function $g \circ \gamma^{-1}$ on $[\gamma(b), \gamma(a)]$. Clearly, $\widetilde{\mathcal{P}}_{N}: \gamma(b)=$ $t_{1}^{(N)}<t_{2}^{(N)}<\cdots<t_{N-1}^{(N)}<t_{N}^{(N)}=\gamma(a), \quad N=1,2, \ldots$, is a sequence of partitions with $\left|\widetilde{\mathcal{P}}_{N}\right| \rightarrow 0$, hence we obtain the last statement.

Proof of Theorem 5.2. First we prove the existence of the limit $\underset{N \rightarrow \infty}{\lim } S(N)$. We have $S(N)=S_{1}(N)+S_{2}(N)$, where

$$
\begin{aligned}
& S_{1}(N):=-\sum_{i=2}^{N-1} y\left(s_{i}^{(N)}, \gamma\left(s_{i}^{(N)}\right)\right) \gamma^{\prime}\left(s_{i}^{(N)}\right)\left(W\left(s_{i+1}^{(N)}, \gamma\left(s_{i}^{(N)}\right)\right)-W\left(s_{i}^{(N)}, \gamma\left(s_{i}^{(N)}\right)\right)\right) \\
& S_{2}(N):=-\sum_{i=2}^{N-1} y\left(s_{i}^{(N)}, \gamma\left(s_{i}^{(N)}\right)\right) \gamma^{\prime}\left(s_{i}^{(N)}\right)^{-1}\left(W\left(s_{i}^{(N)}, \gamma\left(s_{i-1}^{(N)}\right)\right)-W\left(s_{i}^{(N)}, \gamma\left(s_{i}^{(N)}\right)\right)\right) .
\end{aligned}
$$

By Lemma 4.1, the existence of the limit $\operatorname{li.i.m.~}_{N \rightarrow \infty} S_{1}(N)$ follows from

$$
\lim _{N, M \rightarrow \infty} \mathrm{E}\left[S_{1}(N) S_{1}(M)\right]=\int_{a}^{b} \gamma(s) \gamma^{\prime}(s)^{2} y(s, \gamma(s))^{2} \mathrm{~d} s
$$

which is a consequence of Lemma 5.3. Similarly, the limit $\underset{N \rightarrow \infty}{\lim .} S_{2}(N)$ exists, since, again by Lemma 5.3 ,

$$
\lim _{N, M \rightarrow \infty} \mathrm{E}\left[S_{2}(N) S_{2}(M)\right]=\int_{\gamma(b)}^{\gamma(a)} \gamma^{-1}(t) y\left(\gamma^{-1}(t), t\right)^{2} \gamma^{\prime}\left(\gamma^{-1}(t)\right)^{-2} \mathrm{~d} t .
$$

Hence we obtain the existence of the limit $\underset{N \rightarrow \infty}{\lim _{N}} S(N)$.

We have

$$
\frac{1}{h} \int_{\Gamma} y(\cdot)\left(W\left(\cdot+h n_{\Gamma}(\cdot)\right)-W(\cdot)\right)=I_{1,1}(h)+I_{2,1}(h)+I(h),
$$


where

$$
\begin{aligned}
I_{1,1}(h):=\frac{1}{h} \int_{a}^{b} y(s, \gamma(s)) \sqrt{1+\gamma^{\prime}(s)^{2}}(W(s+h \psi(s), \gamma(s))-W(s, \gamma(s))) \mathrm{d} s, \\
I_{2,1}(h):=\frac{1}{h} \int_{a}^{b} y(s, \gamma(s)) \sqrt{1+\gamma^{\prime}(s)^{2}}\left(W\left(s, \gamma(s)+h \sqrt{1-\psi(s)^{2}}\right)-W(s, \gamma(s))\right) \mathrm{d} s, \\
I(h):=\frac{1}{h} \int_{a}^{b} y(s, \gamma(s)) \sqrt{1+\gamma^{\prime}(s)^{2}}\left[W\left(s+h \psi(s), \gamma(s)+h \sqrt{1-\psi(s)^{2}}\right)+W(s, \gamma(s))\right. \\
\left.-W\left(s, \gamma(s)+h \sqrt{1-\psi(s)^{2}}\right)-W(s+h \psi(s), \gamma(s))\right] \mathrm{d} s,
\end{aligned}
$$

hence the statement of the theorem will follow from

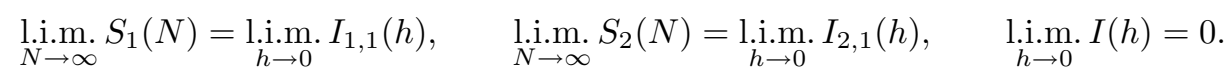

The aim of the following discussion is to prove

$$
\underset{N \rightarrow \infty}{\lim .} S_{1}(N) \stackrel{\text { A }}{=} \underset{h \rightarrow 0}{\operatorname{iim} .} I_{1,2}(h) \stackrel{\text { B }}{=} \underset{h \rightarrow 0}{\operatorname{iim} .} I_{1,1}(h),
$$

where

$$
I_{1,2}(h):=\frac{1}{h} \int_{a}^{b} y(s, \gamma(s)) \psi^{\prime}(s) \sqrt{1+\gamma^{\prime}(s)^{2}}(W(s+h, \gamma(s))-W(s, \gamma(s))) \mathrm{d} s .
$$

In order to prove equality $\mathbf{A}$, we will show that for a sequence $h_{N} \downarrow 0$, we have $S_{1}(N)-I_{1,2}\left(h_{N}\right) \stackrel{\mathrm{L}^{2}}{\longrightarrow} 0$, that is,

$$
\lim _{N \rightarrow \infty} \mathrm{E}\left(S_{1}(N)-I_{1,2}\left(h_{N}\right)\right)^{2}=0 .
$$

Lemma 5.3 implies

$\lim _{N \rightarrow \infty} \mathrm{E} S_{1}(N)^{2}=\lim _{N \rightarrow \infty} \mathrm{E} I_{1,2}\left(h_{N}\right)^{2}=\lim _{N \rightarrow \infty} \mathrm{E}\left[S_{1}(N) I_{1,2}\left(h_{N}\right)\right]=\int_{a}^{b} y(s, \gamma(s))^{2} \gamma^{\prime}(s)^{2} \gamma(s) \mathrm{d} s$,

which completes the proof of equality $\mathbf{A}$.

In order to prove equality $\mathbf{B}$, first we note that the function $\psi$ can be continuously extended to $[a, b]$. Consider the functions

$$
\begin{aligned}
& \psi_{N}(s):=\sum_{i=2}^{N} \psi\left(s_{i}^{(N)}\right)_{\left[s_{i-1}^{(N)}, s_{i}^{(N)}\right)}(s), \quad s \in[a, b) ; \\
& \psi_{N}(b):=\lim _{s \uparrow b} \psi_{N}(s), \quad N=1,2, \ldots
\end{aligned}
$$

Then $\left\|\psi_{N}-\psi\right\| \rightarrow 0$, where $\|\cdot\|$ denotes the supremum norm. To obtain equality $\mathbf{B}$, it is sufficient to show that 
(i) $\lim _{N \rightarrow \infty} \limsup _{h \rightarrow 0} \mathrm{E}\left(I_{1,2}(h)-I_{1,3}(h, N)\right)^{2}=0$,

(ii) $\lim _{h \rightarrow 0} \mathrm{E}\left(I_{1,3}(h, N)-I_{1,4}(h, N)\right)^{2}=0 \quad$ for all $N=1,2 \ldots$,

(iii) $\lim _{N \rightarrow \infty} \limsup _{h \rightarrow 0} \mathrm{E}\left(I_{1,4}(h, N)-I_{1,1}(h)\right)^{2}=0$,

where

$$
\begin{aligned}
& I_{1,3}(h, N):=\frac{1}{h} \int_{a}^{b} y(s, \gamma(s)) \psi_{N}(s) \sqrt{1+\gamma^{\prime}(s)^{2}}(W(s+h, \gamma(s))-W(s, \gamma(s))) \mathrm{d} s, \\
& I_{1,4}(h, N):=\frac{1}{h} \int_{a}^{b} y(s, \gamma(s)) \sqrt{1+\gamma^{\prime}(s)^{2}}\left(W\left(s+h \psi_{N}(s), \gamma(s)\right)-W(s, \gamma(s))\right) \mathrm{d} s .
\end{aligned}
$$

We have

$$
\begin{array}{r}
I_{1,2}(h)-I_{1,3}(h, N)=\frac{1}{h} \int_{a}^{b} y(s, \gamma(s))\left(\psi(s)-\psi_{N}(s)\right) \sqrt{1+\gamma^{\prime}(s)^{2}} \\
(W(s+h, \gamma(s))-W(s, \gamma(s))) \mathrm{d} s .
\end{array}
$$

Applying Lemma 5.3 we obtain

$$
\begin{aligned}
& \mathrm{E}\left(I_{1,2}(h)-I_{1,3}(h, N)\right)^{2} \\
& \leqslant \frac{\left\|\psi-\psi_{N}\right\|^{2}}{h^{2}} \int_{a}^{b} \int_{a}^{b}|y(s, \gamma(s)) y(t, \gamma(t))| \sqrt{\left(1+\gamma^{\prime}(s)^{2}\right)\left(1+\gamma^{\prime}(t)^{2}\right)} \\
& \quad \mathrm{E}[(W(s+h, \gamma(s))-W(s, \gamma(s)))(W(t+h, \gamma(t))-W(t, \gamma(t)))] \mathrm{d} s \mathrm{~d} t \\
& \leqslant c\left\|\psi-\psi_{N}\right\|^{2}
\end{aligned}
$$

with some constant $c$ depending only on the functions $y$ and $\psi$, thus we conclude (i).

Moreover,

$$
\begin{aligned}
& \lim _{h \rightarrow 0} I_{1,3}(h, N) \\
& =\underset{h \rightarrow 0}{\lim . m} \frac{1}{h} \sum_{i=2}^{N} \psi\left(s_{i}^{(N)}\right) \int_{s_{i-1}^{(N)}}^{s_{i}^{(N)}} y(s, \gamma(s)) \sqrt{1+\gamma^{\prime}(s)^{2}}(W(s+h, \gamma(s))-W(s, \gamma(s))) \mathrm{d} s \\
& =\underset{h \rightarrow 0}{\lim .} \frac{1}{h} \sum_{i=2}^{N} \int_{s_{i-1}^{(N)}}^{s_{i}^{(N)}} y(s, \gamma(s)) \sqrt{1+\gamma^{\prime}(s)^{2}}\left(W\left(s+h \psi\left(s_{i}^{(N)}\right), \gamma(s)\right)-W(s, \gamma(s))\right) \mathrm{d} s \\
& =\underset{h \rightarrow 0}{\lim . \ln } I_{1,4}(h, N) \text {, }
\end{aligned}
$$

for all $N=1,2, \ldots$, which implies (ii).

Further, we have 


$$
\begin{aligned}
& I_{1,4}(h, N)-I_{1,1}(h)= \\
& \frac{1}{h} \int_{a}^{b} y(s, \gamma(s)) \sqrt{1+\gamma^{\prime}(s)^{2}}\left(W\left(s+h \psi_{N}(s), \gamma(s)\right)-W(s+h \psi(s), \gamma(s))\right) \mathrm{d} s
\end{aligned}
$$

hence

$$
\begin{aligned}
& \mathrm{E}\left(I_{1,4}(h, N)-I_{1,1}(h)\right)^{2}= \\
& \quad \frac{1}{h^{2}} \int_{a}^{b} \int_{a}^{b} y(s, \gamma(s)) y(t, \gamma(t)) \sqrt{\left(1+\gamma^{\prime}(s)^{2}\right)\left(1+\gamma^{\prime}(t)^{2}\right)} E_{N}(s, t) \mathrm{d} s \mathrm{~d} t,
\end{aligned}
$$

where

$$
\begin{aligned}
E_{N}(s, t):=\mathrm{E}[ & \left(W\left(s+h \psi_{N}(s), \gamma(s)\right)-W(s+h \psi(s), \gamma(s))\right) \\
& \left.\times\left(W\left(t+h \psi_{N}(t), \gamma(t)\right)-W(t+h \psi(t), \gamma(t))\right)\right] .
\end{aligned}
$$

If $u \in \mathbb{R}$ is between the numbers $s+h \psi_{N}(s)$ and $s+h \psi(s)$, then

$$
u \in\left[s+h \psi_{N}(s)-h\left\|\psi_{N}-\psi\right\|, s+h \psi_{N}(s)+h\left\|\psi_{N}-\psi\right\|\right],
$$

hence

$$
\begin{aligned}
& \mid E_{N}(s, t) \mid \\
& \leqslant \mathrm{E}\left[\left(W\left(s+h \psi_{N}(s)+h\left\|\psi_{N}-\psi\right\|, \gamma(s)\right)-W\left(s+h \psi_{N}(s)-h\left\|\psi_{N}-\psi\right\|, \gamma(s)\right)\right)\right. \\
&\left.\left(W\left(t+h \psi_{N}(t)+h\left\|\psi_{N}-\psi\right\|, \gamma(t)\right)-W\left(t+h \psi(t)-h\left\|\psi_{N}-\psi\right\|, \gamma(t)\right)\right)\right] \\
&=\sum_{i, j=1}^{N} \mathrm{E}\left[\left(W\left(s+h \psi_{N}\left(s_{i}^{(N)}\right)+h\left\|\psi_{N}-\psi\right\|, \gamma(s)\right)-W\left(s+h \psi_{N}\left(s_{i}^{(N)}\right)-h\left\|\psi_{N}-\psi\right\|, \gamma(s)\right)\right)\right. \\
&\left.\quad\left(W\left(t+h \psi_{N}\left(s_{j}^{(N)}\right)+h\left\|\psi_{N}-\psi\right\|, \gamma(t)\right)-W\left(t+h \psi\left(s_{j}^{(N)}\right)-h\left\|\psi_{N}-\psi\right\|, \gamma(t)\right)\right)\right] \\
& {\left[s_{i-1}^{\left.(N), s_{i}^{(N)}\right] \times\left[s_{j-1}^{(N)}, s_{j}^{(N)}\right]}(s, t) .\right.}
\end{aligned}
$$

Consequently,

$$
\begin{aligned}
& \mathrm{E}\left(I_{1,4}(h, N)-I_{1,1}(h)\right)^{2} \\
& \quad \leqslant \frac{1}{h^{2}} \sup _{u \in[a, b]}\left[y(u, \gamma(u))^{2}\left(1+\gamma^{\prime}(u)^{2}\right) \gamma(u)\right] \sum_{i, j=1}^{N} \int_{s_{i-1}^{(N)}}^{s_{i}^{(N)}} \int_{s_{j-1}^{(N)}}^{s_{j}^{(N)}} L_{i, j}^{(N)}(s, t) \mathrm{d} s \mathrm{~d} t,
\end{aligned}
$$


where

$$
\begin{array}{r}
L_{i, j}^{(N)}(s, t):=\lambda\left(\left[s+h \psi_{N}\left(s_{i}^{(N)}\right)-h\left\|\psi_{N}-\psi\right\|, s+h \psi_{N}\left(s_{i}^{(N)}\right)+h\left\|\psi_{N}-\psi\right\|\right]\right] \\
\left.\bigcap\left[t+h \psi_{N}\left(s_{j}^{(N)}\right)-h\left\|\psi_{N}-\psi\right\|, t+h \psi_{N}\left(s_{j}^{(N)}\right)+h\left\|\psi_{N}-\psi\right\|\right]\right),
\end{array}
$$

and $\lambda$ denotes the Lebesgue measure. It is easy to see that

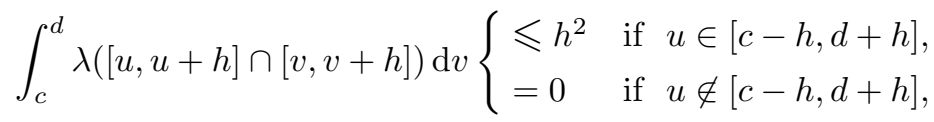

thus

$$
\int_{s_{j-1}^{(N)}}^{s_{j}^{(N)}} L_{i, j}^{(N)}(s, t) \mathrm{d} t\left\{\begin{array}{rr}
\leqslant 4 h^{2}\left\|\psi_{N}-\psi\right\|^{2} & \text { if } s \in\left[s_{j-1}^{(N)}+h\left(\psi\left(s_{j}^{(N)}\right)-\psi_{N}\left(s_{i}^{(N)}\right)-2\left\|\psi_{N}-\psi\right\|\right),\right. \\
& \left.s_{j}^{(N)}+h\left(\psi\left(s_{j}^{(N)}\right)-\psi_{N}\left(s_{i}^{(N)}\right)+2\left\|\psi_{N}-\psi\right\|\right)\right]
\end{array}\right.
$$

Hence

$$
\begin{aligned}
& \frac{1}{h^{2}} \sum_{i, j=1}^{N} \int_{s_{i-1}^{(N)}}^{s_{i}^{(N)}} \int_{s_{j-1}^{(N)}}^{s_{j}^{(N)}} L_{i, j}^{(N)}(s, t) \mathrm{d} s \mathrm{~d} t \\
& \leqslant 4\left\|\psi_{N}-\psi\right\|^{2} \sum_{i, j=1}^{N} \lambda\left([ s _ { i - 1 } ^ { ( N ) } , s _ { i } ^ { ( N ) } ] \cap \left[s_{j-1}^{(N)}+h\left(\psi\left(s_{j}^{(N)}\right)-\psi_{N}\left(s_{i}^{(N)}\right)-2\left\|\psi_{N}-\psi\right\|\right),\right.\right. \\
& \left.\left.s_{j}^{(N)}+h\left(\psi\left(s_{j}^{(N)}\right)-\psi_{N}\left(s_{i}^{(N)}\right)+2\left\|\psi_{N}-\psi\right\|\right)\right]\right) \\
& \leqslant 4\left\|\psi_{N}-\psi\right\|^{2} \sum_{j=1}^{N}\left(4 h\left\|\psi_{N}-\psi\right\|+s_{j}^{(N)}-s_{j-1}^{(N)}\right) \\
& =4\left\|\psi_{N}-\psi\right\|^{2}\left(4 h\left\|\psi_{N}-\psi\right\|+b-a\right) \rightarrow 4\left\|\psi_{N}-\psi\right\|^{2}(b-a) \quad \text { as } h \rightarrow 0,
\end{aligned}
$$

thus

$$
\mathrm{E}\left(I_{1,4}(h, N)-I_{1,1}(h)\right)^{2} \leqslant c\left\|\psi-\psi_{N}\right\|^{2},
$$

with some constant $c$ depending only on the functions $y$ and $\psi$, thus we obtain (iii), and the proof of the equality $\mathbf{B}$ is complete.

Now, let us deal with $S_{2}(N)$. We have

$$
\begin{aligned}
S_{2}(N)=-\sum_{i=2}^{N-1} y\left(\gamma^{-1}\left(t_{N+1-i}^{(N)}\right), t_{N+1-i}^{(N)}\right) \gamma^{\prime}\left(\gamma^{-1}\left(t_{N+1-i}^{(N)}\right)\right)^{-1} \\
\left(W\left(\gamma^{-1}\left(t_{N+1-i}^{(N)}\right), t_{n+2-i}^{(N)}\right)-W\left(\gamma^{-1}\left(t_{i}^{(N)}\right), t_{N+1-i}^{(N)}\right)\right),
\end{aligned}
$$


where $t_{i}^{(N)}=\gamma\left(s_{N+1-i}^{(N)}\right)$, and $\widetilde{\mathcal{P}}_{N}: \gamma(a)=t_{1}^{(N)}<t_{2}^{(N)}<\cdots<t_{N-1}^{(N)}<t_{N}^{(N)}=\gamma(b)$, $N=1,2, \ldots$, is a sequence of partitions with $\left|\widetilde{\mathcal{P}}_{N}\right| \rightarrow 0$. Applying the same procedure for the curve $\widetilde{\gamma}(t):=\gamma^{-1}(t)$ on the interval $[\gamma(b), \gamma(a)]$ as before, we obtain

$$
\underset{N \rightarrow \infty}{\lim .} S_{2}(N)=\underset{h \rightarrow 0}{\operatorname{li} . m .} \widetilde{I}_{2,1}(h),
$$

where

$$
\widetilde{I}_{2,1}(h):=\frac{1}{h} \int_{\gamma(b)}^{\gamma(a)} y\left(\gamma^{-1}(t), t\right) \sqrt{1+\widetilde{\gamma}^{\prime}(t)^{2}}\left(W\left(\gamma^{-1}(t), t+h \widetilde{\psi}(t)\right)-W\left(\gamma^{-1}(t), t\right)\right) \mathrm{d} t,
$$

and

$$
\sqrt{1+\widetilde{\gamma}^{\prime}(t)^{2}}=\sqrt{1+\frac{1}{\gamma^{\prime}\left(\gamma^{-1}(t)\right)^{2}}}, \quad \widetilde{\psi}(t)=-\frac{\widetilde{\gamma}^{\prime}(t)}{\sqrt{1+\widetilde{\gamma}^{\prime}(t)^{2}}}=\frac{1}{\sqrt{1+\gamma^{\prime}\left(\gamma^{-1}(t)\right)^{2}}} .
$$

By Lemma 4.6 we easily obtain $\widetilde{I}_{2,1}(h)=I_{2,1}(h)$, hence we conclude $\underset{N \rightarrow \infty}{\lim .} S_{2}(N)=$ $\lim _{h \rightarrow 0} I_{2,1}(h)$.

Finally we have

$$
\mathrm{E} I(h)^{2}=\frac{1}{h} \int_{a}^{b} \int_{a}^{b} y(s, \gamma(s)) y(t, \gamma(t)) \sqrt{\left(1+\gamma^{\prime}(s)^{2}\right)\left(1+\gamma^{\prime}(t)^{2}\right)} E(s, t) \mathrm{d} s \mathrm{~d} t,
$$

where

$$
\begin{aligned}
E(s, t):=\mathrm{E}[\{ & W\left(s+h \psi(s), \gamma(s)+h \sqrt{1-\psi(s)^{2}}\right)-W(s+h \psi(s), \gamma(s)) \\
& \left.-W\left(s, \gamma(s)+h \sqrt{1-\psi(s)^{2}}\right)+W(s, \gamma(s))\right\} \\
& \left\{W\left(t+h \psi(t), \gamma(t)+h \sqrt{1-\psi(t)^{2}}\right)-W(t+h \psi(t), \gamma(t))\right. \\
& \left.\left.-W\left(t, \gamma(t)+h \sqrt{1-\psi(t)^{2}}\right)+W(t, \gamma(t))\right\}\right] .
\end{aligned}
$$

Clearly

$$
\begin{aligned}
E(s, t) & =\lambda\left([s, s+h \psi(s)] \times\left[\gamma(s), \gamma(s)+h \sqrt{1-\psi(s)^{2}}\right]\right. \\
& \left.\bigcap[t, t+h \psi(t)] \times\left[\gamma(t), \gamma(t)+h \sqrt{1-\psi(t)^{2}}\right]\right) \\
\leqslant & \frac{h^{2}}{4}\{[s, s+h \psi(s)] \cap[t, t+h \psi(t)] \neq \emptyset\}(s, t) \\
\leqslant & \frac{h^{2}}{4}\{|s-t| \leqslant h\}
\end{aligned}
$$


and

$$
\int_{a}^{b} \int_{a}^{b}\{|s-t| \leqslant h\}(s, t) \mathrm{d} s \mathrm{~d} t=2 h(b-a)-h^{2}
$$

hence

$$
\mathrm{E} I(h)^{2} \leqslant \frac{2 h(b-a)-h^{2}}{4} \sup _{s \in[a, b]}\left[y(s, \gamma(s))^{2}\left(1+\gamma^{\prime}(s)^{2}\right)\right] \rightarrow 0 \quad \text { as } h \rightarrow 0,
$$

thus we obtain that $\underset{h \rightarrow 0}{\lim . m} I(h)=0$.

\section{References}

[1] Arató, M. (1982). Linear stochastic systems with constant coefficients. A statistical approach. (Lecture Notes in Control and Inf., vol. 45, 309 pp.) Berlin: Springer-Verlag. (In Russian, Moscow: Nauka, 1989).

[2] Arató, N.M. (1997). Mean estimation of Brownian and Ornstein-Uhlenbeck sheets. Theor. Veroyatn. Primen. 42, 375-376.

[3] Arató, N.M. (1997). Mean estimation of Brownian sheet. Computers Math. Applic. 33, 13-25.

[4] Ash, R.B. and Gardner, M.F. (1975). Topics in Stochastic Processes. Academic Press, New York, San Francisco, London.

[5] Baran, S., Pap, G. and Zuijlen, M.v. (2000). Estimation of the mean of stationary and nonstationary Ornstein-Uhlenbeck processes and sheets. Report No. 0003, University of Nijmegen, The Netherlands, 2000. To appear in Computers Math. Applic.

[6] Billingsley, P. (1986). Convergence of probability measures. John Wiley \& Sons, New York.

[7] Kuo, Hui-Hsiung (1975). Gaussian Measures in Banach Spaces, SpringerVerlag, Berlin, Heidelberg, New York. 